1999-05-01

\title{
Postlaunch sensor verification and calibration of the NASA Scatterometer
}

David G. Long

david_long@byu.edu

Wu-yang Tsai

James E. Graf

Carroll Winn

James N. Huddleston

See next page for additional authors

Follow this and additional works at: https://scholarsarchive.byu.edu/facpub

Part of the Electrical and Computer Engineering Commons

\section{Original Publication Citation}

Tsai, W. -Y, et al. "Postlaunch Sensor Verification and Calibration of the NASA Scatterometer."

Geoscience and Remote Sensing, IEEE Transactions on 37.3 (1999): 1517-42

\section{BYU ScholarsArchive Citation}

Long, David G.; Tsai, Wu-yang; Graf, James E.; Winn, Carroll; Huddleston, James N.; Dunbar, R. Scott; Freilich, Michael H.; Wentz, Frank J.; and Jones, W. Linwood, "Postlaunch sensor verification and calibration of the NASA Scatterometer" (1999). Faculty Publications. 623.

https://scholarsarchive.byu.edu/facpub/623

This Peer-Reviewed Article is brought to you for free and open access by BYU ScholarsArchive. It has been accepted for inclusion in Faculty Publications by an authorized administrator of BYU ScholarsArchive. For more information, please contact ellen_amatangelo@byu.edu. 


\section{Authors}

David G. Long, Wu-yang Tsai, James E. Graf, Carroll Winn, James N. Huddleston, R. Scott Dunbar, Michael H. Freilich, Frank J. Wentz, and W. Linwood Jones 


\title{
Postlaunch Sensor Verification and Calibration of the NASA Scatterometer
}

\author{
Wu-yang Tsai, James E. Graf, Carroll Winn, James N. Huddleston, Scott Dunbar, Michael H. Freilich, \\ Frank J. Wentz, David G. Long, Senior Member, IEEE, and W. Linwood Jones, Fellow, IEEE
}

\begin{abstract}
Scatterometer instruments are active microwave sensors that transmit a series of microwave pulses and measure the returned echo power to determine the normalized radar backscattering cross section (sigma-0) of the ocean surface from which the speed and direction of near-surface ocean winds are derived. The NASA Scatterometer (NSCAT) was launched on board the ADEOS spacecraft in August 1996 and returned ten months of high-quality data before the failure of the ADEOS spacecraft terminated the data stream in June 1997.

The purpose of this paper is to provide an overview of the NSCAT instrument and sigma-0 computation and to describe the process and the results of an intensive postlaunch verification, calibration, and validation effort. This process encompassed the functional and performance verification of the flight instrument, the sigma-0 computation algorithms, the science data processing system, and the analysis of the sigma-0 and wind products. The calibration process included the radiometric calibration of NSCAT using both engineering telemetry and science data and the radiometric beam balance of all eight antenna beams using both open ocean and uniform land targets. Finally, brief summaries of the construction of the NSCAT geophysical model function and the verification and validation of the wind products will be presented.

The key results of this paper are as follows: The NSCAT instrument was shown to function properly and all functional parameters were within their predicted ranges. The instrument electronics subsystems were very stable and all of the key parameters, such as transmit power, receiver gain, and bandpass filter responses, were shown to be stable to within $0.1 \mathrm{~dB}$. The science data processing system was thoroughly verified and the sigma-0 computation error was shown to be less than $0.1 \mathrm{~dB}$. All eight antenna beams were radiometrically balanced, using natural targets, to an estimated accuracy of about $0.3 \mathrm{~dB}$. Finally, a new model function, called NSCAT-1, was constructed and used to produce wind products. The wind products were statistically verified using ECMWF wind fields and were validated using NDBC buoy measurements. Overall, we believe that NSCAT generated high-quality wind products with wind speed and direction accuracies that met the science requirements.
\end{abstract}

Index Terms-Calibration, NSCAT, scatterometer, sea, winds.

Manuscript received April 15, 1998; revised January 21, 1999. The work was supported by the Jet Propulsion Laboratory, California Institute of Technology, under a contract with the National Aeronautics and Science Administration

W. Tsai, J. E. Graf, C. Winn, J. N. Huddleston, and S. Dunbar are with the Jet Propulsion Laboratory, California Institute of Technology, Pasadena, CA 91109 USA.

M. H. Freilich is with the College of Oceanic and Atmospheric Sciences, Oregon State University, Corvallis, OR 97331 USA.

F. J. Wentz is with the Remote Sensing Systems, Santa Rosa, CA 95404 USA.

D. G. Long is with the Electrical and Computer Engineering Department, Brigham Young University, Provo, UT 84601 USA.

W. L. Jones is with the Central Florida Remote Sensing Laboratory, Electrical and Computer Engineering Department, University of Central Florida, Orlando, FL 32816 USA.

Publisher Item Identifier S 0196-2892(99)03568-8.

\section{INTRODUCTION}

$\mathbf{S}$ PACEBORNE scatterometers are active microwave radar instruments designed to acquire near-simultaneous, spatially collocated measurements of the normalized radar backscattering cross section (sigma-0) of the ocean surface from several azimuth and/or incidence angles. The value of sigma- 0 is a function of incidence angle, wind speed, and azimuth (horizontal) angle between the microwave radiation and the wind direction. A quantitative model (geophysical model function) of the relationship between sigma-0 and the wind vector, the measurement geometry, and polarization, is then used to retrieve both the wind speed and direction over the ocean.

In the past, several spaceborne scatterometers have been flown on Skylab, Seasat-A, ERS-1, and ERS-2. A Kuband scatterometer, the NASA Scatterometer (NSCAT), was launched on board the Japanese Advanced Earth Observation Satellite (ADEOS) on August 17, 1996. It was turned on on September 10, 1996, and operated almost flawlessly until June 30, 1997, when ADEOS lost electrical energy output from the solar panels and the spacecraft, with NSCAT on board, was lost [1].

During the 40-week timespan, NSCAT, with its wide swath, demonstrated its usefulness by providing accurate, frequent, global measurements of sigma-0 (over land, ice, and ocean) and near-surface wind velocity (over the ice-free oceans). The near-surface wind vector measurements are used in ocean, atmosphere, and climate research, such as wind-driven upper ocean circulation research, marine meteorology research, air-, sea-, and coupled-climate research, and El Niño, monsoon, storm, and hurricane studies. The wind vector measurements were also used in operational weather applications, such as numerical weather forecasting, ocean ship routing, and storm and hurricane tracking. The sigma- 0 measurements were used for ice detection and classification, land usage monitoring, and vegetation classification. Initial results were reported in a NSCAT Science Working Team meeting on November 10-14, 1997 [2].

The purpose of this paper is to provide an overview of the NSCAT instrument and to describe the process and the results of the intensive postlaunch verification, calibration, and validation efforts conducted at the Jet Propulsion Laboratory after NSCAT was turned on in mid-September, 1996. The verification process encompassed the functional and performance verification of the flight instrument, determination of the spacecraft attitude, verification of the sigma-0 computation algorithms, verification of the science processing system, 


\section{NASA SCATTDROMPTER (NSCAT) ON ADDOS}

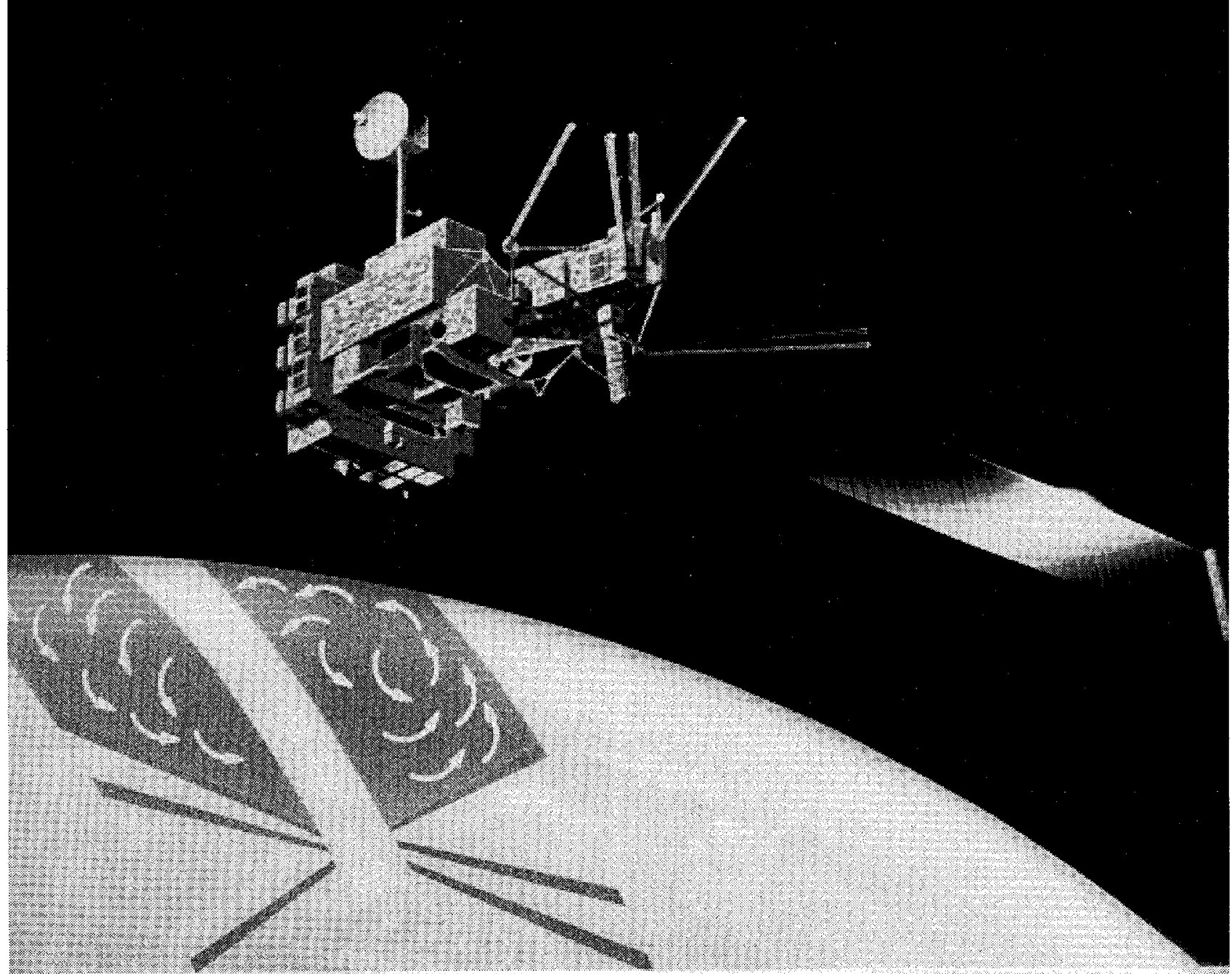

Fig. 1. NASA Scatterometer on ADEOS spacecraft. NSCAT is located at the front part of the spacecraft. The structure was built to accommodate six sets of stick antennas, RF electronics, and digital electronics.

and determination of the quality of the sigma- 0 and wind products. The calibration process included the radiometric calibration of NSCAT using: 1) engineering telemetry and science data, 2) a calibration ground station (CGS), and 3) the radiometric balance of all eight antenna beams, using natural targets and the CGS. Finally, the validation process involved the validation of the NSCAT wind accuracy using in-situ measurements.

\section{OVERVIEW OF THE NSCAT INSTRUMENT AND GROUND PROCESSING SYSTEM}

In order to understand the overall postlaunch verification and calibration process, some background about the NSCAT system design and processing system is required. In this section, we will provide a brief summary of key NSCAT system design features and the ground processing system. A detailed description of the NSCAT system can be found in [3].

\section{A. Overview of the NSCAT Instrument System}

A special tower-like mounting structure was installed at the front of the ADEOS spacecraft to accommodate the NSCAT antennas due to their stringent field-of-view requirements (see Fig. 1). Each side of the swath (with respect to the spacecraft ground track) is illuminated by four antenna beams from different azimuth angles (the fore and aft beams are vertically polarized, while the midbeam is dual-polarized) (see Fig. 2). The backscattered signals are Doppler-filtered using an onboard digital signal processor to produce 25 equally spaced high-resolution $(25 \mathrm{~km})$ sigma- 0 cells simultaneously. The data collected from these four antenna beams (measured from three different azimuth angles) allows for the retrieval of the wind speed and direction over a $600-\mathrm{km}$ swath on each side of the spacecraft ground track.

The flight instrument consists of four major subsystems (see Fig. 3): the radio-frequency subsystem (RFS), the antenna 


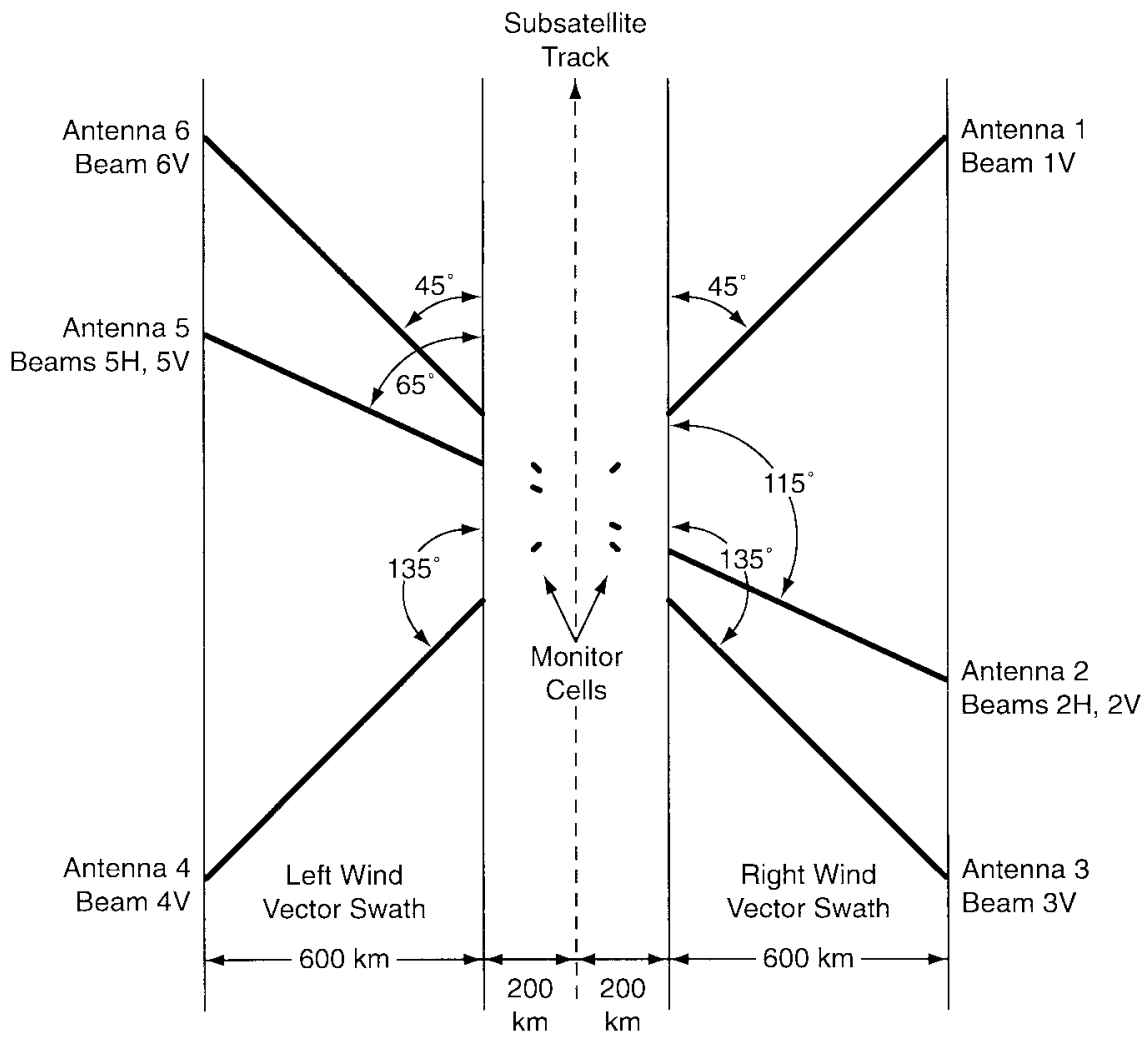

Fig. 2. NSCAT antenna illumination pattern on the ground. NSCAT has four antenna beams on each side of the spacecraft nadir track (subsatellite track). Aside from the regular 12 sigma- 0 cell measurements for each antenna within the wind vector swath, there is also a monitor cell for each antenna beam, located roughly at $10^{\circ}$ incidence angle. The monitor cell is used to monitor the stability of the instrument electronics, since the radar backscattering cross section is insensitive to wind speed and direction at this incidence angle.

subsystem, the digital subsystem (DSS), and the mechani$\mathrm{cal} /$ thermal subsystem (MTS). The function of the RFS is to generate the transmit pulses and route them to the antenna subsystem through a waveguide-switch matrix to the selected antenna beam; to receive the return signal, down-convert and pass the signal to the DSS; and to calibrate the receiver gain using a noise source. The transmit pulse has a 5-ms pulse width and has a pulse repetition frequency of $62 \mathrm{~Hz}$ with each measurement consisting of a sequence of 25 transmit pulses. The transmit pulses are amplified by a high-voltage traveling wave tube amplifier (TWTA) to a peak power of about $110 \mathrm{~W}$. The received signals are downconverted, passed through four crystal filters, and further downconverted to baseband before being input to the DSS. In order to achieve high accuracy in the measurement of the echo power, the RFS has two internal, well calibrated, and highly stable calibrators: a transmit power monitor (for measuring the transmit power) and a noise source (for measuring the receiver gain). Furthermore, to minimize the receiver gain variation over the orbit, a temperature compensation loop was built in to the RFS receiver system.

The antenna subsystem consists of six identical, dualpolarized fan beam antennas. Each antenna is made up of two separate slotted waveguide arrays. While all antennas are dual-polarized, only the vertical polarization is used for antennas 1, 3, 4, and 6 (see Fig. 2). Both vertical and horizontal polarizations are used for antennas 2 and 5. To achieve the desired fan beam illumination pattern on the earth's surface, the antennas were designed to be $10 \mathrm{ft}$ long, 2.5 in wide, and 4 in deep. The antennas are comprised of graphite-epoxy horns and thin-wall aluminum waveguides. Each antenna produces a fan beam with a $28^{\circ} 3-\mathrm{dB}$ beamwidth in elevation (along-beam direction) and a $0.4^{\circ}$ beamwidth in azimuth (crossbeam direction). The MTS provides antenna stow (launch restraint) and deployment mechanisms and the structural support for the RFS and DSS. It also provides a stable thermal environment, maintaining the instrument in a tight temperature range $\left(<2{ }^{\circ} \mathrm{C}\right)$ via a passive control scheme employing louvers.

The DSS consists of two processors: a command and control processor (CP) and a digital Doppler processor (DDP). The $\mathrm{CP}$ performs the control functions for instrument operations, including receiving commands from the spacecraft and collecting telemetry data. In addition, the $\mathrm{CP}$ also computes certain processing parameters for the Doppler processor. The onboard DDP is used to perform Doppler beam-sharpening of the antenna beam in order to reduce the data rate, improve the coregistration accuracy of signals from the four antenna beams on each side of the ground track, and allow for the accurate calibration of the backscattered power measurements.

\section{B. Overview of the Science Data Processing System}

The ground data system for NSCAT consisted of the data delivery system and the science data processing system 


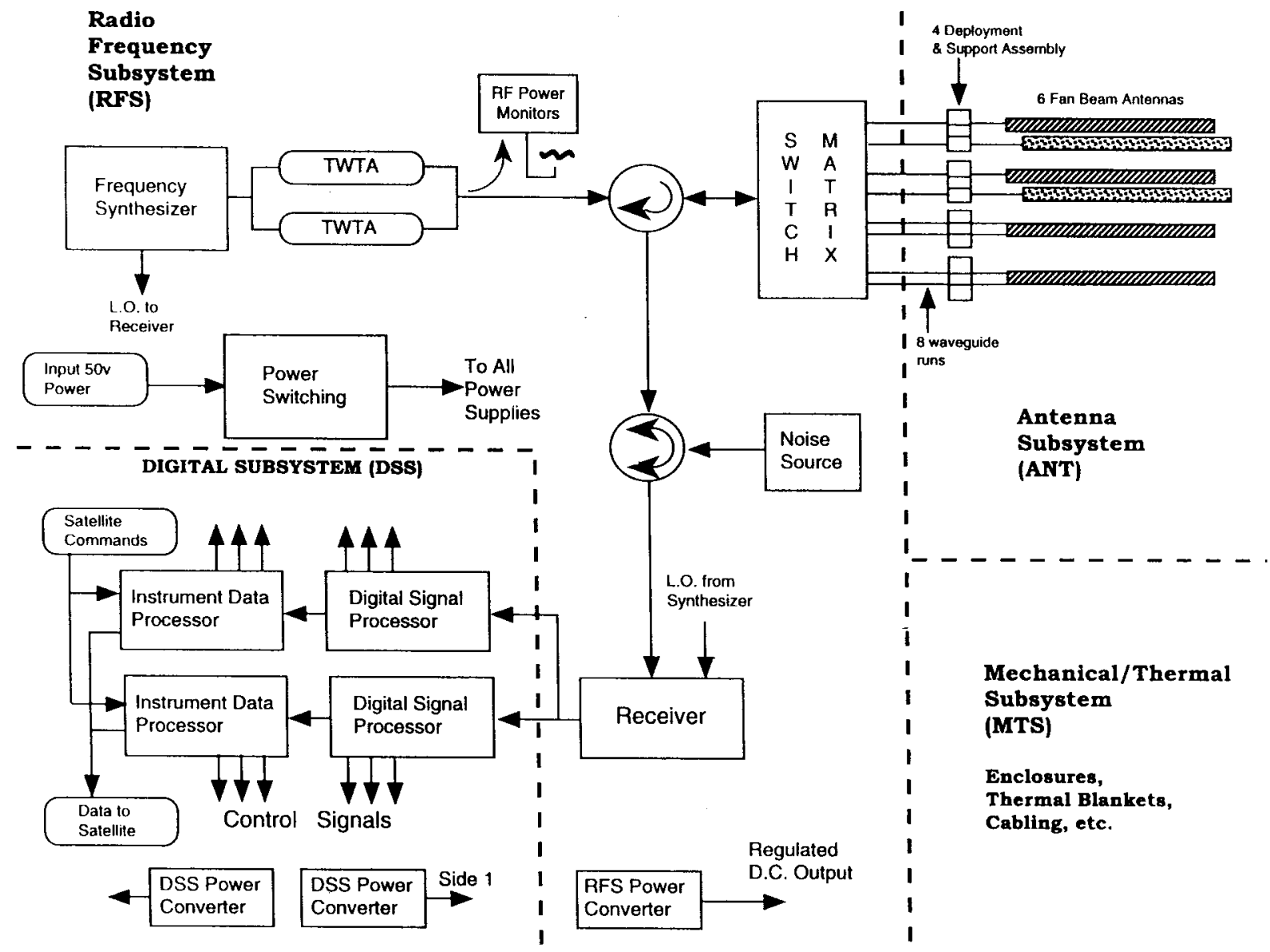

Fig. 3. NSCAT instrument functional block diagram.

(SDPS). The SDPS performed data ingestion, geometric collocation, sigma-0 computation, wind product generation, and product archive and distribution. The calculation of vector winds from raw NSCAT data requires: 1) calculating earth-located sigma-0 values for each antenna beam and collocating ocean sigma-0 measurements from beams with different azimuths, 2) estimating a set of probable vector winds from the collocated sigma- 0 measurements through inversion of the geophysical model function and the statistical solution of an over determined system, and 3) selecting a unique wind vector from the set of probable wind vectors. Due to the stringent radiometric accuracy requirements, the sigma-0 computation algorithms for NSCAT are quite sophisticated and their computation accuracy needed to be verified postlaunch.

\section{OvERVIEW OF END-TO-END SigMA-0 COMPUTATION, InSTRUMENT PERFoRMANCE, AND CALIBRATION REQUIREMENTS}

\section{A. Overview of the Sigma-0 Computation}

The NSCAT instrument transmits electromagnetic pulses to the ocean surface and makes an echo signal plus noise measurement and a noise only measurement. The radar equation is then used to compute sigma- 0 . We can write a general formulation of the radar equation in the following form [4]:

$$
\begin{aligned}
P_{r}^{S} & =P_{r}^{S+N}-P_{r}^{N} \\
& =\frac{P_{t} G_{r} \lambda^{2}}{L_{s}(4 \pi)^{3}} \int d^{2} \bar{r} \frac{G_{a}^{2}(\bar{r}) \sigma_{0}(\bar{r})}{R^{4}(\bar{r})} F(\cdots)
\end{aligned}
$$

where $P_{r}^{S}$ is the measured signal power, $P_{r}^{S+N}$ is the measured signal-plus-noise power, $P_{r}^{N}$ is the measured noise-only power, $\sigma_{\circ}$ is the normalized radar backscattering cross section, $R$ is the slant range, $L_{s}$ is the two-way platform waveguide loss, $P_{t}$ is the transmit power, $G_{a}$ is the antenna gain, $G_{r}$ is the RFS receiver gain, and $\lambda$ is the transmit wavelength. The effective filter function $F(\cdots)$ is a function of the range gate timing, signal time delay, cell location, and slant range. The two-dimensional integration is over all of the footprint of the total two-dimensional ground area of a given sigma- 0 cell.

In general, $\sigma_{o}$ inside the integral is a function of ground location. However, because we are interested in the average value of $\sigma_{o}$ over a given resolution cell, we can express (1) in the following form:

$$
\sigma_{0}=K P_{r}^{S} \frac{(4 \pi)^{3} L_{s}}{P_{t} G_{r} A_{\mathrm{eff}} \lambda^{2}}\left(\frac{R^{4}}{G_{a}^{2}}\right)_{c}
$$

where $A_{\text {eff }}$ is the $3-\mathrm{dB}$ cell area, and $(\cdots)_{c}$ are the radar parameters evaluated at the center of the resolution cell. The correction factor (termed " $K$-factor") takes into account the antenna pattern variation within a resolution cell, digital 


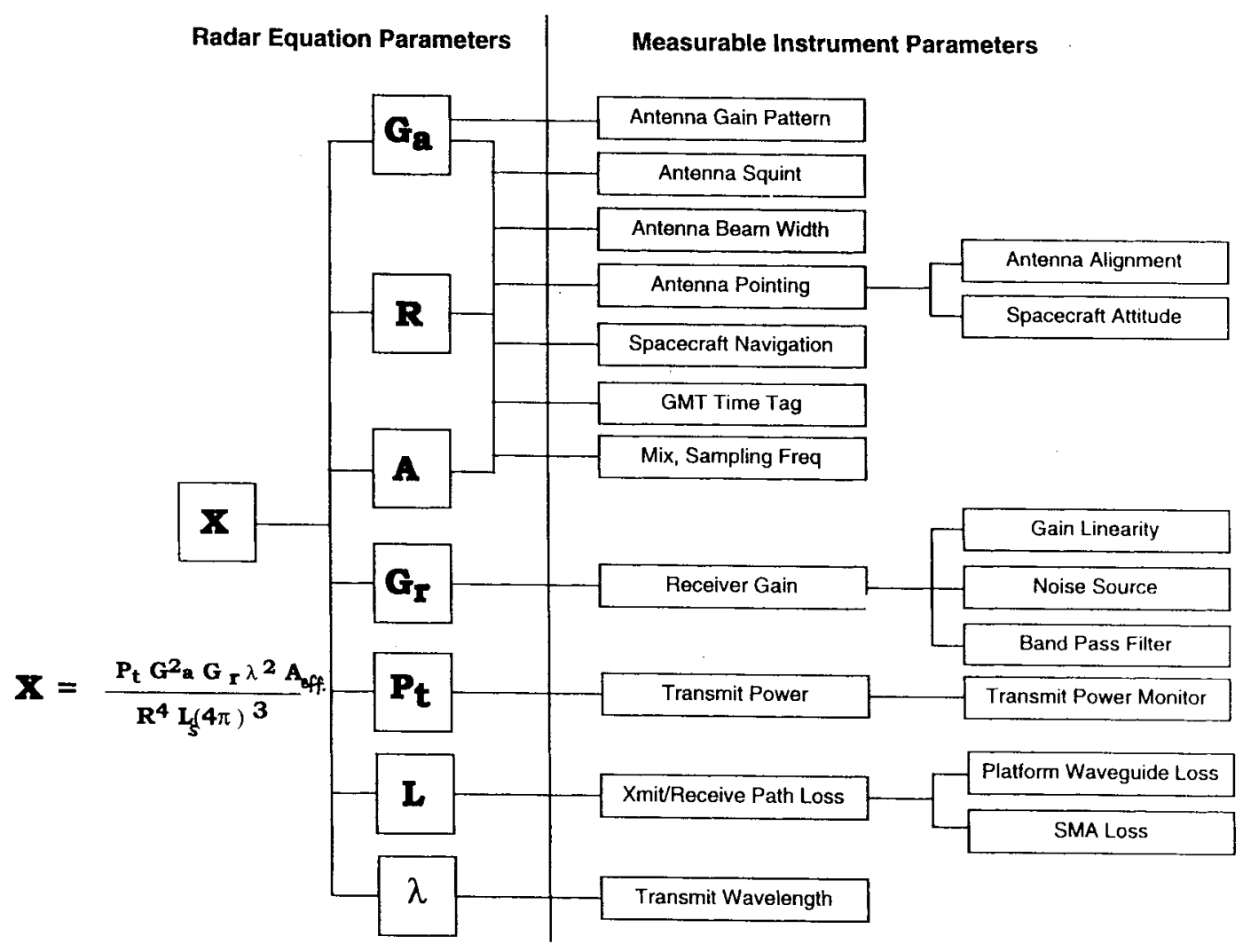

Fig. 4. Relationship between radar parameters and measurable instrument parameters.

processor effects, transmit pulse shape, and range gating timing. $K$-factor can be expressed in the following form:

$$
K=\frac{A_{\mathrm{eff} \sigma_{0}}\left(\frac{G_{a}^{2}}{R^{4}}\right)_{c}}{\int d^{2} \bar{r} \frac{G_{a}^{2}(\bar{r}) \sigma_{0}(\bar{r})}{R^{4}(\bar{r})} F(\cdots)} .
$$

This formula was implemented in software, and calculations with NSCAT instrument and orbital parameters were carried out prelaunch and provided to the SDPS in tabular form for the sigma-0 computation [4].

The computation of sigma- 0 is further complicated by the fact that some of the radar parameters given in (2) are not directly measurable physical quantities, but are derived from measurable instrument and spacecraft parameters as shown in Fig. 4. Algorithms for computing these relationships were tested extensively prelaunch and needed to be verified postlaunch.

\section{B. Overview of Instrument Performance and Calibration Requirements}

Instrument design and calibration requirements were levied to meet the science requirements [6]. The coverage requirement of obtaining data for $90 \%$ of the open ocean (in two days) was achieved by designing the instrument to have a $600-\mathrm{km}$ swath on each side of the spacecraft nadir track. The $50-\mathrm{km}$ wind vector cell resolution requirement was met by Doppler beam sharpening of the antenna beam to produce $25-\mathrm{km}$ sigma-0 resolution cells. These sigma- 0 measurements were then combined to form $50-\mathrm{km}$ resolution wind vector cells.

The wind speed accuracy requirement is $2 \mathrm{~m} / \mathrm{s}$ root-meansquared (rms) error for wind speeds from $3-20 \mathrm{~m} / \mathrm{s}$, and $10 \%$ of the wind speed for speeds between 20 and $30 \mathrm{~m} / \mathrm{s}$. The wind direction error is required to be less than $20 \mathrm{rms}$ for the ambiguity closest to the true wind direction (NSCAT typically generates four possible wind vector solutions, called ambiguities, for each wind vector cell). In order to meet these requirements, we needed to impose stringent performance and calibration requirements on NSCAT. The key performance requirements are: 1) the signal-to-noise ratio (SNR) shall be larger than $-8 \mathrm{~dB}$, for $3 \mathrm{~m} / \mathrm{s}$ winds and 2) the antenna pattern, beam pointing, transmit power, receiver gain, and bandpass filter response need to be stable and repeatable, with a combined rms variation of less than $0.5 \mathrm{~dB}, 1$-sigma, over $8 \mathrm{~min}$. Extensive prelaunch tests were performed showing that the instrument did indeed meet these requirements (see Table I). One of the key postlaunch verification and calibration tasks was to verify, using mission telemetry and sigma-0 analysis, that the science requirements were met.

\section{Postlaunch Sensor Verification AND CALIBRATION APPROACHES}

For a given radar design, the accuracy of the retrieved wind speed and direction depends on the accuracy of the measured sigma- 0 values and the uncertainty of the geophysical model function. The verification and calibration process involved the following key steps. 
TABLE I

Summary of NSCAT Prelaunch Calibration Results

\begin{tabular}{|c|c|c|c|c|}
\hline \multirow{2}{*}{ Calibration Parameter } & \multicolumn{2}{|c|}{ Prclaunch Calibration Bias } & \multicolumn{2}{|c|}{ Time-Varying Calibration Error } \\
\hline & Parameter Error & $\sigma_{0}$ Error & Parameter Error & $\sigma_{0}$ Error \\
\hline Antenna Peak Gain & $0.35 \mathrm{~dB}$ & $0.7 \mathrm{~dB}$ (2-way) & \multirow[t]{2}{*}{$0.10 \mathrm{~dB}$} & \multirow[t]{2}{*}{$0.20 \mathrm{~dB}$ (2-way) } \\
\hline Antenna Broad Beam Pattern & $0.02 \mathrm{~dB} / \mathrm{dB}$ & $0.40 \mathrm{~dB} \max (2-w a y)$ & & \\
\hline Antenna Beam Width & $0.015^{\circ}$ & $0.16 \mathrm{~dB}$ & $0.005^{\circ}$ & $0.05 \mathrm{~dB}$ \\
\hline Antenna Squint & $0.05^{\circ}$ & $0.09 \mathrm{~dB} \max$ & $0.007^{\circ}$ & $0.01 \mathrm{~dB}$ \\
\hline Antenna Pointing & $0.05^{\circ}$ each axis & $0.05 \mathrm{~dB}$ & $0.08^{\circ}$ each axis & $0.13 \mathrm{~dB}$ \\
\hline Spacecraft Navigation & N/A & $0.00 \mathrm{~dB}$ & $1 \mathrm{~km}, 2 \mathrm{~m} / \mathrm{s}$ each axis & $0.00 \mathrm{~dB}$ \\
\hline GMT Time Tag & $\mathrm{N} / \mathrm{A}$ & $0.00 \mathrm{~dB}$ & $0.70 \mathrm{sec}$ & $0.00 \mathrm{~dB}$ \\
\hline Mixing Frequency & $200.00 \mathrm{~Hz}$ & $0.03 \mathrm{~dB}$ & $<200.0 \mathrm{~Hz}$ & $0.03 \mathrm{~dB}$ \\
\hline Receiver Gain & $0.17 \mathrm{~dB}$ & $0.17 \mathrm{~dB}$ & $0.10 \mathrm{~dB}$ & $0.10 \mathrm{~dB}$ \\
\hline Transmit Power & $0.35 \mathrm{~dB}$ & $0.35 \mathrm{~dB}$ & $<0.10 \mathrm{~dB}$ & $0.10 \mathrm{~dB}$ \\
\hline System Loss & $0.24 \mathrm{~dB}$ & $0.48 \mathrm{~dB}(2$-way) & $0.05 \mathrm{~dB}$ & $0.10 \mathrm{~dB}$ (2-way) \\
\hline Transmit Wavelength & $1.00 \mathrm{MHz}$ & $0.00 \mathrm{~dB}$ & $<1.00 \mathrm{MHz}$ & $0.00 \mathrm{~dB}$ \\
\hline RSS Sums & \multicolumn{2}{|c|}{ Prelaunch Bias $=1.03 \mathrm{~dB}$} & \multicolumn{2}{|c|}{$\mathrm{Kpr}=0.31 \mathrm{~dB}$} \\
\hline
\end{tabular}

Postlaunch Bias Removal Uncertainty: $0.2 \mathrm{~dB}$ Beam-to-beam, $0.5 \mathrm{~dB}$ Absolute

Step 1-Instrument Functional and Performance Verification: Immediately after instrument turn on, we needed to verify that the antennas were deployed properly, the electronic subsystems were functioning properly, and the key instrument calibration parameters were stable over time. This required the use of the engineering, science, and spacecraft telemetry. We also needed to verify the stability of the key instrument calibration parameters, such as transmit power, receiver gain, and crystal filter frequency response.

Step 2-Science Data Processing System (SDPS) Verification: The computation algorithms contained in the SDPS are an end-to-end realization of the total NSCAT measurement process starting from raw data acquisition, through sigma0 computation, to wind vector retrieval, and data product generation. Therefore, the verification of the SDPS involved functional verification, calibration parameter and table verification, end-to-end sigma- 0 computation algorithm verification, and sigma-0 product verification.

Step 3-Sensor Calibration: The retrieved wind vector accuracy depends on both the relative and absolute calibration accuracy of the scatterometer system. The relative radiometric calibration accuracy affects the wind vector accuracy, while good absolute radiometric calibration accuracy ensures the capability for accurate determination of the Ku-band geophysical model function. Major calibration issues addressed here are the beam pointing of the antennas and the beam-to-beam radiometric balance.

Step 4-Construction of a Geophysical Model Function: Estimation of wind velocity from sigma-0 involves the inversion of a geophysical model function, which is a functional relationship between sigma-0 and the wind vectors, measurement geometry, and polarization. A method, using numerical weather product (NWP) wind fields, special sensor microwave/imager (SSM/I) data, and wind field analysis for the construction of a new model function is presented later.

Step 5-Wind Product Verification and Validation: The wind product verification involved the identification of sys- tematic errors in the NSCAT winds through comparisons between NSCAT data and selected statistics of operational surface wind analyses. The wind product validation involved the determination of the wind speed and direction accuracy of the interim NSCAT vector wind data based on detailed comparisons with independent, high-quality, open-ocean buoy measurements.

\section{NSCAT MisSION OpERATION TIMELINE}

NSCAT is one of the six scientific instruments on board the ADEOS spacecraft. The NSCAT antennas were deployed about 12 hours after launch. The electronic subsystem was turned on on September 10, 1996, and the engineering checkout was conducted within the first five and half days (see Fig. 5). The first day was dedicated to functional checkout, and the next two days were spent collecting calibration and performance data in continuous calibration mode (CCM). The instrument was then commanded to operate in wind observation mode (WOM) to collect science data for sigma0 and wind measurements. Special binning constants were uplinked at the beginning of the WOM period to operate the instrument in a special high sigma-0 resolution mode for two days: one day each at 12.5 and $6.25 \mathrm{~km}$ resolutions. Finally, a new set of binning constants, using the updated ADEOS orbital parameters, were uplinked to operate the instrument in WOM at the nominal sigma- 0 resolution of $25 \mathrm{~km}$.

NSCAT operated in nominal WOM from September 15, 1996 to June 30, 1997, with the exception of a two-day period from January 15-16, 1997, during which NSCAT collected higher resolution $(12.5 \mathrm{~km})$ sigma-0 data. As mentioned before, NSCAT ceased operation on June 30, 1997, due to the failure of the ADEOS solar panel [1].

\section{Postlaunch Sensor Verification}

The sensor verification activities described in this section include the verification of all components of the instrument and processor systems which affect the determination of sigma- 0 . 


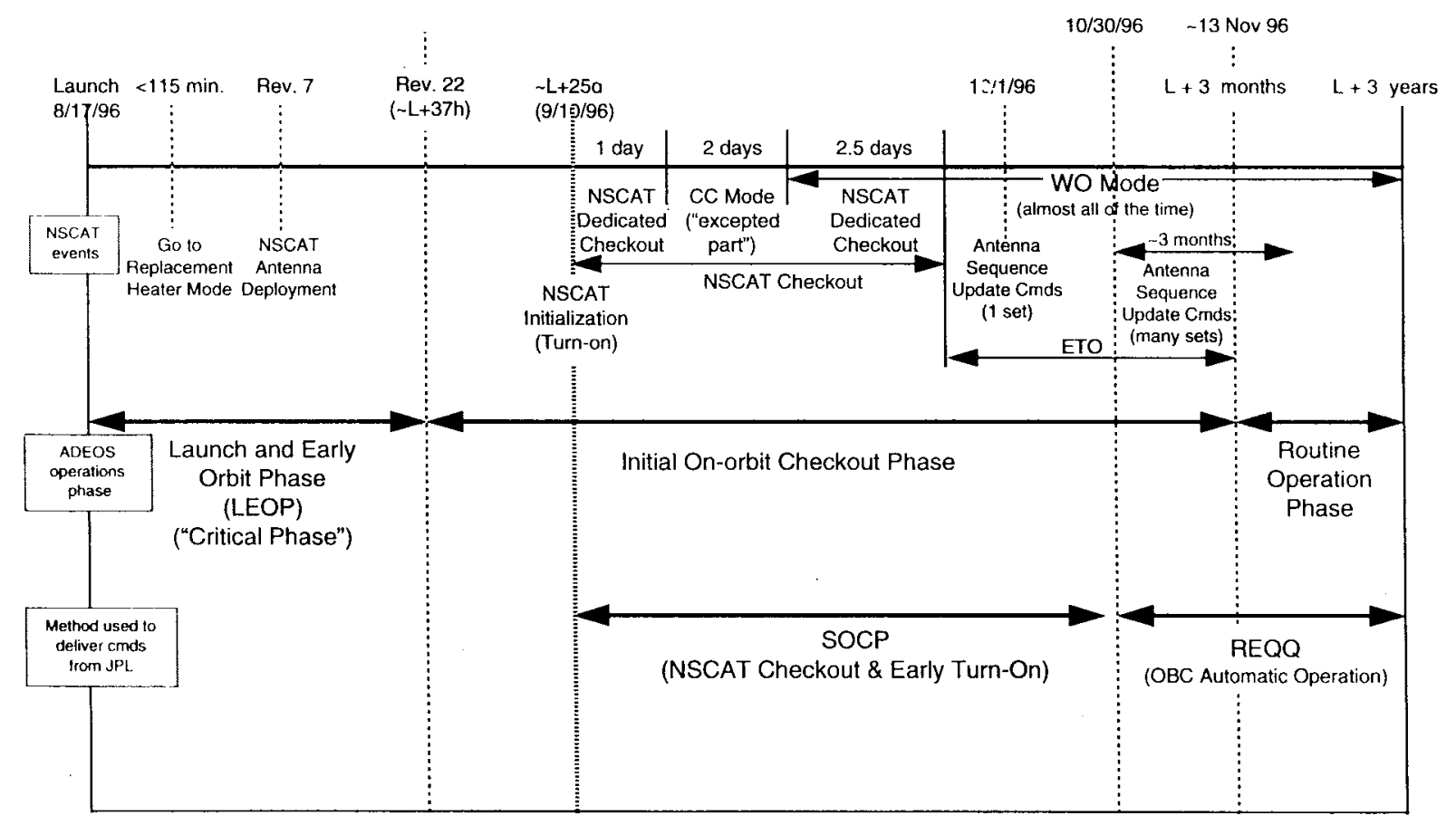

Fig. 5. NSCAT mission timeline.

\section{A. Instrument Functional Verification}

As a part of NSCAT mission operations, sophisticated software was developed for automated instrument functional verification, limit checking, and performance and calibration parameter monitoring. The instrument was first checked and verified during the initial turn on and later during normal operations in WOM.

The instrument was operated in every mode during the initial check-out, and all functional and performance parameters were monitored continuously. The following parameters were limit-checked daily: DIU (DC/DC interface unit) and DSS voltages; main bus current; Traveling-wave-tube (TWT) body current, body regulator voltage, and ion pump current; spacecraft attitude and altitude; IPM (input power monitor for the TWTA) and transmit power monitor (TPM) measurements; dc offsets for all four receiver channels; gain and noise figures for all four receiver channels and all four local oscillators (LO's); channel powers, signal-plus-noise and noise-only cell powers; orbit timer; and temperatures for the ASM (antenna switch matrix), crystal filters, DIU, DSS, HVPS (high voltage power supply for the TWTA), IPM, LNA (low noise amplifier), noise source, REU (RF electronics unit), STALO (stable local oscillator), TPM, and TWTA. All these parameters were shown to be very stable and within expected limits.

The following errors were also checked for on a daily basis: CSB (circulator switch bank)/beam mismatch, invalid mode, receiver protect in wrong state, HVPS backup commanded, TWTA undervoltage trip, TWTA overcurrent trip, TWTA body overcurrent trip, lack of startup requirements, error queue full, fault counter change, ULM (UHF loop module) and SLM (synthesizer loop module) unlocked, TWT trip override enabled, TWT monitor disabled, TWT monitor HVPS (highvoltage power supply) shutdown disabled, and unexpected cycle counter. There were also no errors during our monitoring period.

Some of the key results of the instrument functional checkout are as follows.

1) Within a given orbit, the temperature variation of the $\mathrm{RF}$ electronics subsystem was less than $2{ }^{\circ} \mathrm{C}$. This is accomplished by the use of louvers and a clear, unobstructed view of deep space.

2) All NSCAT operational modes were exercised successfully.

3) The digital subsystem was verified using debug mode data (as compared to the prelaunch test data) which used digital signals of known frequencies and amplitudes.

4) All functional parameters, such as currents, voltages, and temperatures were within safety and predicted limits.

5) The housekeeping engineering telemetry indicated that the antennas were deployed properly. Using sigma0 measurements, the locations of small islands were computed and they agreed with map locations to within $25 \mathrm{~km}$ (the accuracy of the determination method).

\section{B. Instrument Performance Verification}

As mentioned in Section II, in order to achieve high calibration accuracy, NSCAT has two built-in calibrators: the transmit power monitor (TPM) and the noise source (NS). These two internal calibrators allow us to frequently monitor the instrument transmit power and receiver gain.

1) Transmit Power Verification: The transmit power is measured by the TPM by summing the power in the 25 pulses transmitted during a given antenna beam transmit sequence. By calculating a running average of these TPM measurements, we were able to demonstrate that the transmit power (over the four-month initial checkout period) was very stable (to within 


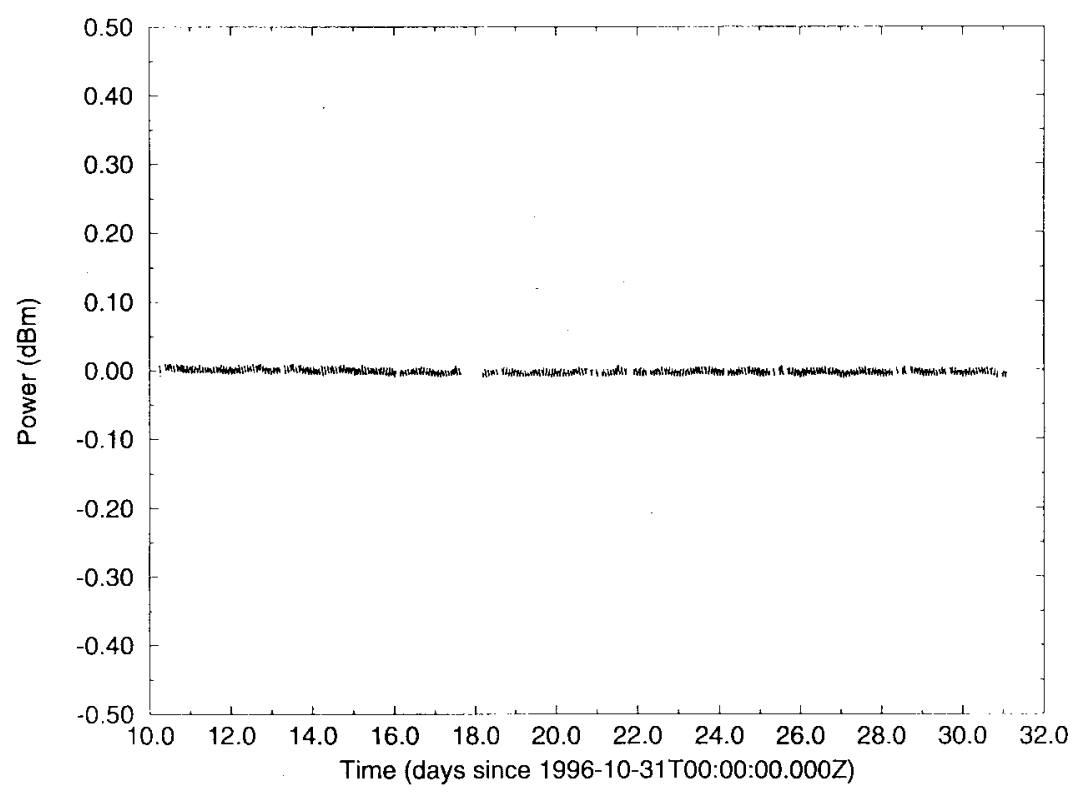

Fig. 6. Plot showing the long-term stability of the transmit power.

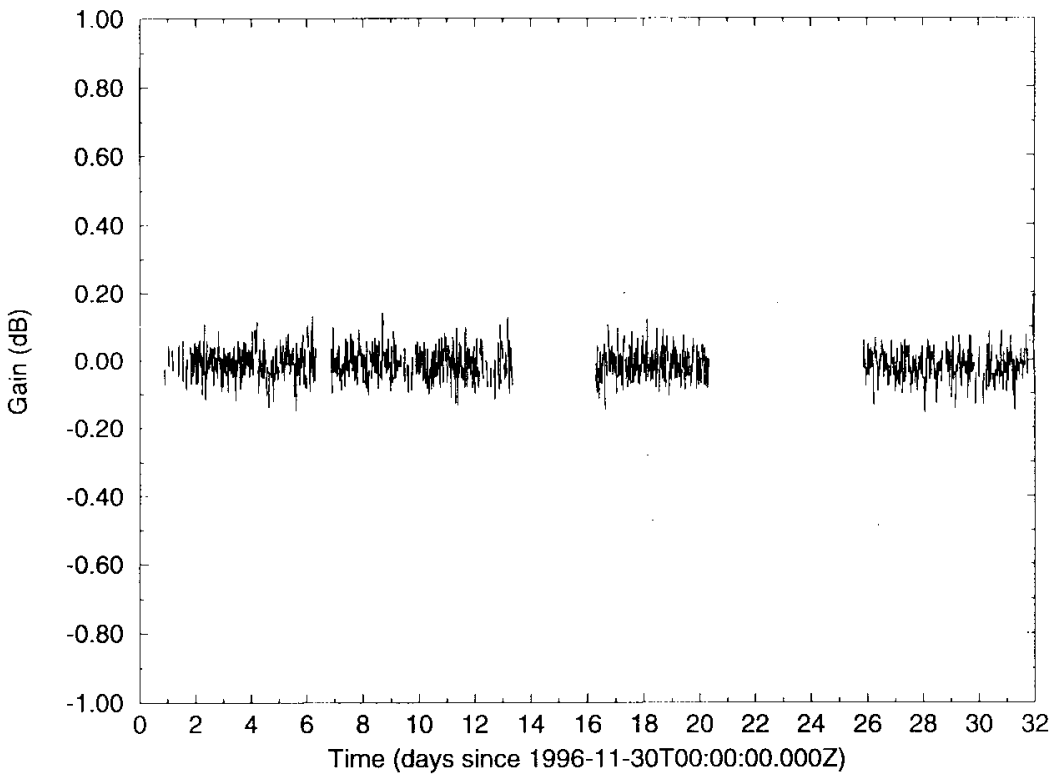

Fig. 7. Plot showing the long-term stability of the receiver gain.

$0.02 \mathrm{~dB}$ ) and the long-term variation (within one orbit) was very small ( $<0.1 \mathrm{~dB})$ (see Fig. 6).

2) Receiver Gain Verification: The receiver gain of the NSCAT instrument was monitored during CCM to demonstrate the short-term (within one orbit and within one day) stability of the 16 combinations (four channels each with four different LO's) of receiver gain. During routine WOM operations, a calibration cycle is conducted once every $8 \mathrm{~min}$ to measure the NSCAT receiver gain.

Furthermore, due to the integration time and receiver bandwidth constraints, the expected receiver gain measurements may have a statistical fluctuation of up to $0.75 \mathrm{~dB}$ peak-topeak [5]. The time scale of the receiver gain variation was determined using the data collected during CCM, where all 16 receiver gains could be computed once every antenna cycle of $3.87 \mathrm{~s}$. We found that the receiver gain variation is very small over one orbit, and that the receiver gain was very stable over a period of, at least, several days. This is due to the fine temperature control of the RFS and due to the implementation of a temperature compensation loop for the receiver gain of the RFS. Using a running average of about 100 calibration cycle measurements (roughly 0.5 day), we can compute the receiver gain to an accuracy of about $0.1 \mathrm{~dB} 3$-sigma for all channels.

The long-term stability of the receiver gain was monitored by using a 200-point running average over the calibration cycle measurements. We were able to demonstrate that, over a period of four months, the receiver gain was stable to within $0.1 \mathrm{~dB}$ 3-sigma (see Fig. 7).

3) Bandpass Filter (BPF) Frequency Response and Spurious Noises Verification: The echo returns of NSCAT are divided 


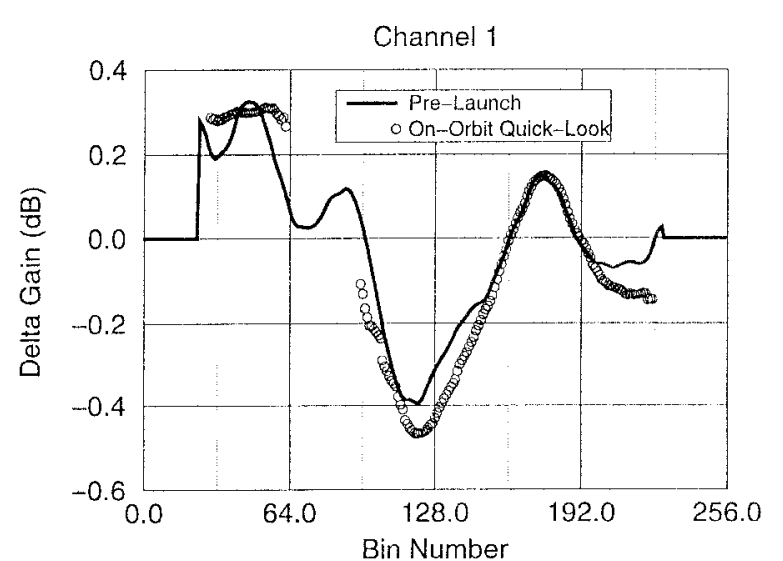

(a)

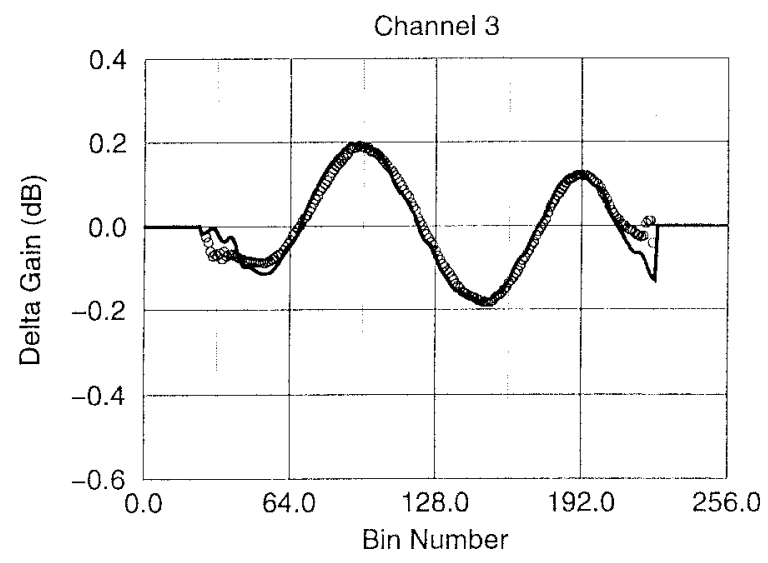

(c)

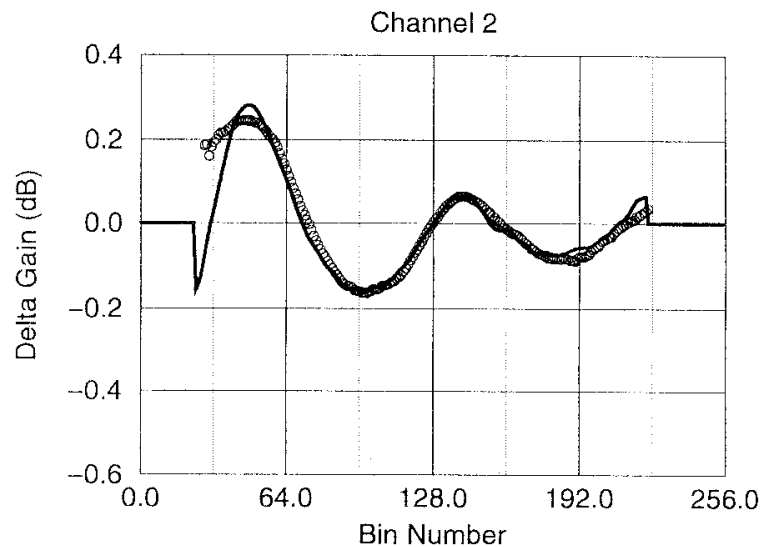

(b)

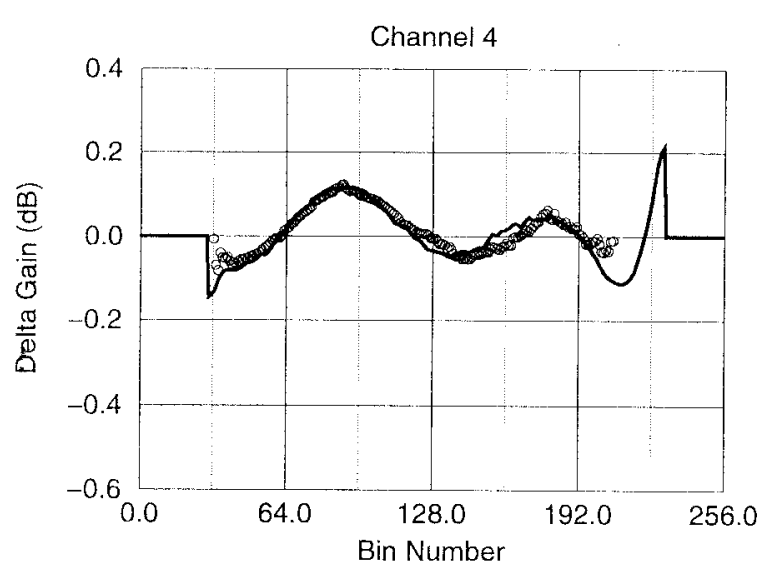

(d)

Fig. 8. Comparison of prelaunch and on-orbit crystal filter frequency response for all four channels. The $x$-axis is the FFT bin number and the $y$-axis (the delta gain) is the bandpass filter gain relative to the average gain for that channel. The FFT bin bandwidth is a function of the channel and is approximately (a) $935 \mathrm{~Hz}$ for channel 1, (b) $515 \mathrm{~Hz}$ for channel 2, (c) $266 \mathrm{~Hz}$ for channel 3, and (d) $238 \mathrm{~Hz}$ for channel 4.

into four frequency channels using four crystal bandpass filters. Due to sigma-0 accuracy requirements, the gain of the BPFO's needed to be stable in time and the response of each channel needed to be calibrated to better than $0.05 \mathrm{~dB}$. One of the key prelaunch instrument calibration tasks was to demonstrate that the BPFO's were stable and to calibrate their frequency response in thermal-vacuum. It was also critical for us to verify, postlaunch, that these filters were stable and their frequency responses remained unchanged.

Algorithms were developed and tested prelaunch to use either the noise-only measurements or the continuous calibration measurements to compute the BPF gain as a function of frequency after launch. The algorithms were based on the fact that the on-board processor generates a sigma- 0 cell measurement by summing over several fast Fourier transform (FFT) bins. The number of bins and the location of the bins varied over the orbit. The algorithms involved accumulating sigma-0 measurement data over a long period of time (two days for CCM and three weeks for WOM), generating an overdetermined set of linear equations in which each cell power is equated to the sum of its FFT bins, and finding a linear least squares solution for the set of equations. In this way, the BPF frequency responses for all four channels is reconstructed to a resolution of one FFT bin.
The agreement between the postlaunch BPF measurements and those obtained prelaunch in thermal-vacuum (shown in Fig. 8) is amazingly good. The variations of the BPF responses were also found to be very small (within the statistical fluctuation of the measurements). The same technique was also used to detect spurious noise. We found that the spurious noises remained small and the frequencies and amplitudes of the spurs were in good agreement with prelaunch measurements.

\section{Science Data Processing System (SDPS) Verification}

Once it was demonstrated that the instrument was functioning properly, was stable over time, and calibrated, the verification of the SDPS began. As was mentioned before, the computation algorithms contained in the SDPS are an endto-end realization of the total NSCAT measurement process, starting from raw data acquisition, through the sigma-0 computation, to wind vector retrieval and data product generation. Therefore, the verification of the SDPS involves the following: SDPS functional verification, calibration parameter and table verification, end-to-end sigma-0 computation algorithm verification, and sigma-0 product verification.

1) Processor System Function Verification: The SDPS was tested extensively prior to launch using realistic simulated data in the ADEOS data formats. A science simulation created raw 
telemetry from a combination of engineering test data and wind field simulations. This data could then be processed endto-end within the SDPS algorithm testbed (ATB). After launch, the ATB was used to process higher-level data products needed during the preliminary calibration period. This allowed for rapid and continuous updates and corrections to be made in the ATB as the data were analyzed. Changes in the ATB would later be propagated to the official data processor.

Aside from processing NSCAT data to generate wind products, the SDPS also implemented quality assurance (QA) procedures to identify and flag data products, at the granule level, which obviously and significantly did not conform to the expected accuracy for the particular product type. Typical QA tasks and products were as follows:

- header record check;

- statistical analyses of each parameter;

- alarm limit checks of parameters;

- histograms of selected variables;

- distribution of various flags;

- global wind vector plots.

In principle, QA was able to detect major problems in wind products before distribution to the science users.

Since sigma- 0 is a derived physical quantity, its computation requires a set of well calibrated constants and tables. Key quantities being checked and updated included antenna deployment angles, antenna gain patterns with an associated definition of coordinate system, and transmit and receive path loss.

2) End-to-End Sigma-0 Computation Algorithm Verification: The NSCAT instrument transmits electromagnetic pulses to the ocean surface and detects the return backscattered power. The radar equation is used to compute sigma- 0 . The computation of sigma- 0 from NSCAT measurements is quite complicated due to the following factors.

- The signal power of an NSCAT measurement is computed from the subtraction of the noise power from the signalplus-noise power measurement. This algorithm is further complicated by the FFT binning in the DSS and the nonflatness of the BPF response.

- The determination of sigma-0 using the radar equation requires a two-dimensional surface integration of the radar parameters. The computation algorithm uses the nominal radar parameters at the center of each sigma-0 cell together with a precalculated correction factor, called $K$-factor, which takes into account the antenna pattern variation within a resolution cell, digital processor effects, range gate timing, and the transmit pulse shape.

- Some of the radar parameters are not directly measurable physical quantities, but they are related to measurable instrument and spacecraft parameters (see Fig. 4). Algorithms for computing these relationships needed to be verified.

Finally, some of the instrument and geometric parameters are computed from the engineering and science telemetry. These algorithms also needed to be verified.

a) Sigma-0 cell location verification: The sigma-0 cell location was calculated using the ADEOS spacecraft ephem- eris and attitude, an earth model, and antenna deployment angles. The first step to verify cell location was to compare the locations of small islands using radar backscatter measurements against detailed land maps. Using this method, we were able to verify that cell locations were correct to less than $25 \mathrm{~km}$.

b) K-factor table verification: As mentioned above, the radar equation correction factor, $K$-factor, is a triple sum of a double spatial integral over the antenna gain pattern and digital filter response (FFT). $K$-factor depends on the binning constants in use by NSCAT, the ADEOS orbital parameters, the antenna gain pattern, the transmit pulse shape, the digital filter response, and the range gate timing. Therefore, it is impractical to calculate this quantity in real time, and a precalculated $K$-factor table needed to be generated. The detailed formulation and tabulation of $K$-factor can be found in [4].

Aside from the careful verification of the theoretical formulation and the numerical computation, a powerful verification of $K$-factor is to show that the sigma-0's from a given cell are continuous across channel boundaries. The on-board processor determines the center frequency and bandwidth of each sigma0 measurement cell using an algorithm and a set of uploadable constants (the binning constants). These center frequencies are varied over the course of an orbit so that each cell maintains a constant cross track distance from the nadir track. The center frequency and bandwidth of each cell is translated into a frequency channel number, one through four, and a range of FFT bins to be summed. The frequency channels overlap such that a cell can always fit entirely within a single channel. Occasionally, a cell moves too close to the edge of its current channel and is moved into the adjacent channel which has a different channel gain and bandpass filter response. Thus, verifying the continuity of sigma- 0 across channel boundary is a powerful tool to verify $K$-factor, because the value of $K$-factor depends on the number of FFT bins within each resolution cell, and the number of FFT bins makes a significant jump at channel boundaries. Thus, if the values of sigma- 0 are continuous across channel boundaries, it is reasonable to assume that $K$-factor correctly compensated for the change in cell bin width. Fig. 9 is a plot of sigma-0 as a function of orbital position, which shows that sigma- 0 is continuous across the channel boundaries (regions highlighted with dark spots).

A final verification of the $K$-factor formulation is to verify that the $K$-factor table is insensitive to spacecraft attitude and altitude variations for the expected range of spacecraft attitude and altitude variation. This study was conducted by Prof. D. Long at Brigham Young University [7].

c) Noise subtraction algorithm verification: The noise subtraction algorithm calculates the signal portion of the received power by subtracting out an estimate of the background noise from each signal-plus-noise measurement. Background noise measurements were made over the entire channel bandwidth (of about $1 \mathrm{MHz}$ ) for a shorter length of time and must be scaled appropriately to estimate the noise power within the narrower bandwidth of the signal-plus-noise measurement made over a longer length of time. The gain of each periodogram bin (the frequency response) is calculated 

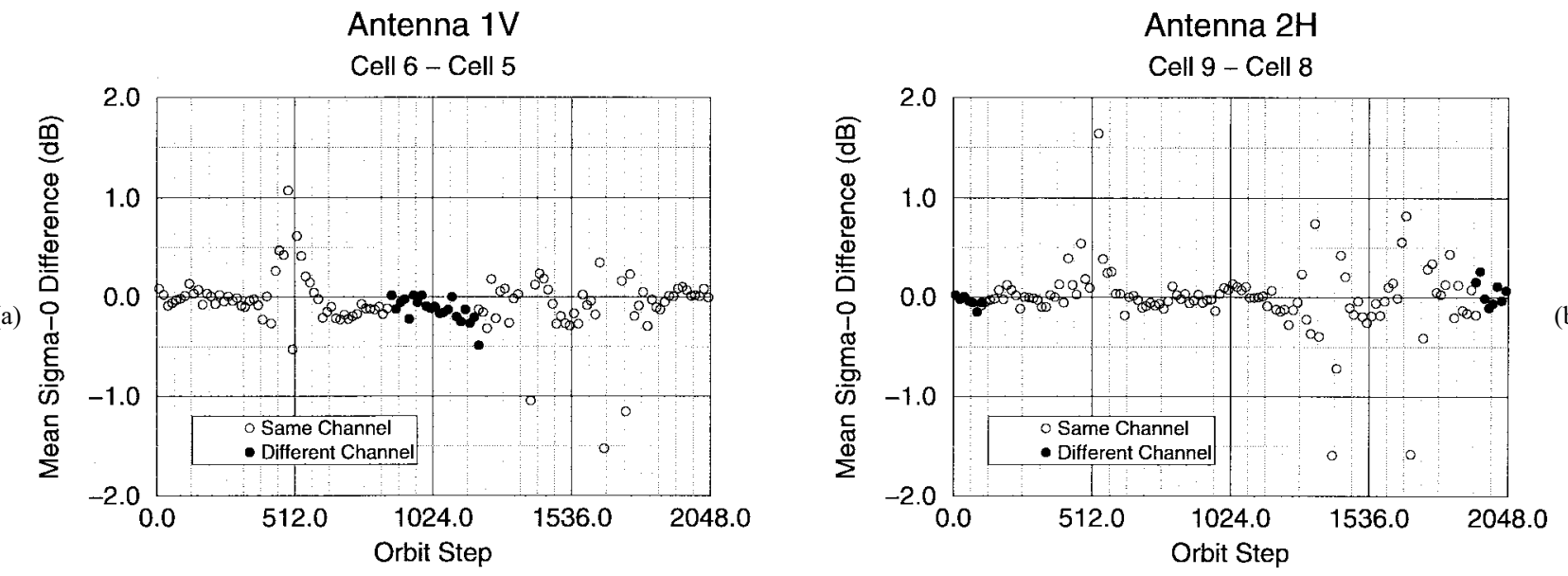

(b)
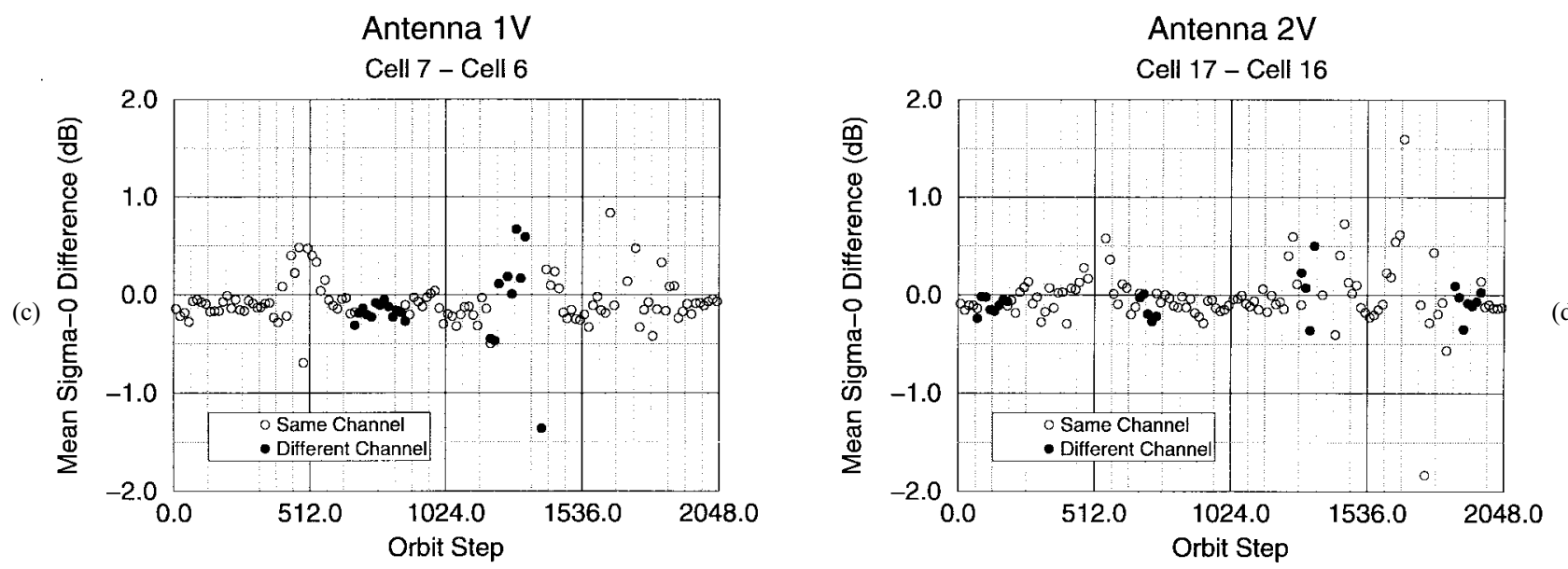

Fig. 9. Mean sigma-0 difference between neighboring cells as a function of orbit position. The dark dots are the sigma-0 difference between cells from two different channels. The $x$-axis is the orbit step number. (Each orbit is divided into 2048 orbit steps, starting and ending at the ascending node (equator crossing), with each orbit step lasting just under $3 \mathrm{~s}$ ). (a) Antenna 1V, cell 6-cell 5; (b) antenna 2H, cell 9-cell 8; (c) antenna 1V, cell 7-cell 6; (d) antenna $2 \mathrm{~V}$; cell $17-$ cell 16.

relative to the gain of the entire channel, and therefore the frequency response only needs to be applied to the signal-plusnoise measurements. The accuracy of this noise subtraction algorithm is especially important for estimating sigma- 0 when SNR is low (wind speed $<3 \mathrm{~m} / \mathrm{s}$ ).

Due to statistical fluctuations, it is possible to calculate negative measured sigma- 0 's for cases in which the signal power is low. One of the methods used to verify the noise subtraction algorithm was to generate the global distribution of negative sigma-0's over both land and ocean (Fig. 10), and to verify that only regions of very low wind speed $(<2 \mathrm{~m} / \mathrm{s})$ over oceans and lakes produce negative values of sigma-0 [8].

Another method used to verify the noise subtraction algorithm was to generate a histogram of sigma- 0 and compare the distribution in the low and negative sigma- 0 regions with that of prelaunch simulation results.

d) g-factor algorithm verification: The $g$-factor is a numerical factor which compensates for range gating effects. Occasionally, the slant range to the earth is such that the entire backscattered echo does not fall into the receiver gate; a portion of the echo is "gated out." This causes the receiver to measure less power than it would have had the entire echo been captured in the range gate. The $g$-factor compensates for the lost portion of the echo. The value of $g$-factor is calculated "on the fly" in the SDPS using spacecraft ephemeris data.

Since the values of $g$-factor depend on the range gate timing, and the range gate timing is different for each of the four channels, the channel boundaries were again used to detect errors in the calculation of $g$-factor. Fig. 9 shows that sigma- 0 is continuous across channel boundaries and therefore demonstrates that $g$-factor is correctly compensating for the range gating effects.

e) Binning algorithm verification: The NSCAT instrument resolves each received echo into 25 narrow bandwidth cells by passing the sampled echo through an FFT and summing given ranges of FFT bins into cells. The location and bandwidth of each cell is calculated by the Doppler binning algorithm. A set of constants, called binning constants, is used by the binning algorithm to control the frequency location, and consequently the physical location, of each sigma- 0 resolution cell. The summation of periodogram bins into cells was performed on orbit by NSCAT and the cell power measurements were downlinked in mission telemetry. The upper and lower frequency of each cell must be determined by the ground data processor in order to calculate the cell's area and to locate the cell on the earth's surface. 


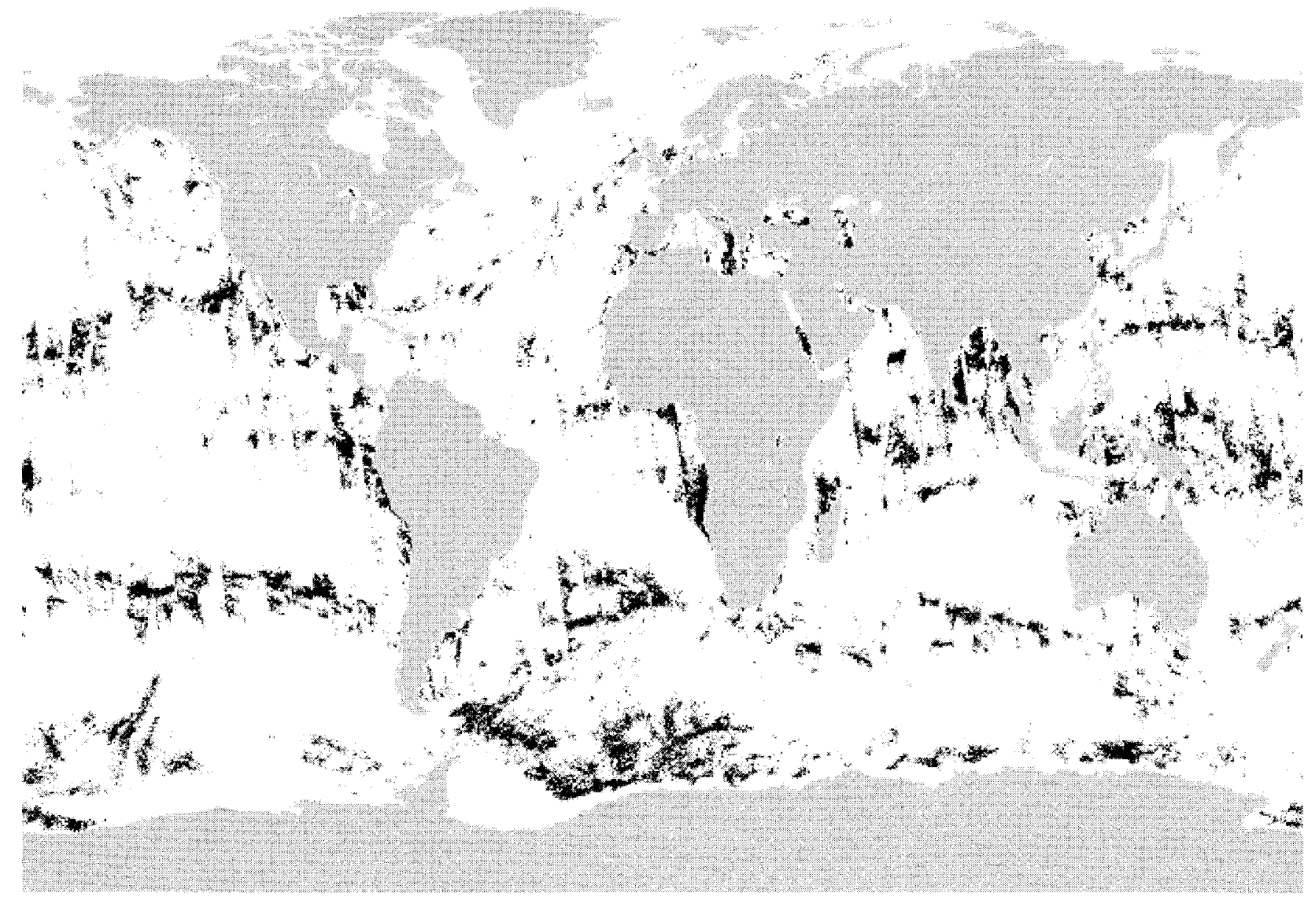

Fig. 10. Global negative sigma-0 map from NSCAT data collected on September 16, 1996.

Extensive testing was performed prelaunch to verify that the binning algorithm used by NSCAT could be exactly reproduced in ground software and would accurately locate the sigma- 0 cells at the desired locations. Postlaunch data was analyzed to verify that the bandwidth of cells was correctly calculated by the SDPS. The channel noise measurement was used to calculate the expected noise power of noiseonly cell measurements. The expected cell noise power was then compared with the noise-only measurement of each cell, after correcting for the bandpass filter ripple effect. No discrepancies were found.

f) Analysis of sigma- 0 products: Since sigma- 0 is a physical quantity, a powerful method to verify the SDPS is to analyze the sigma- 0 products to see whether they satisfy physical constraints. Several sigma-0 analysis methods are described below.

A powerful check on the noise subtraction algorithm was to plot the location of negative sigma-0's over land and ocean (see Fig. 10). Negative sigma-0 can occur in regions where the mean sigma- 0 is expected to be low, due to the statistical nature of the power measurements and the noise subtraction calculation. It was observed that only regions which are clearly identified as lakes had negative sigma- 0 . Also noted was that, over the ocean, the regions with negative sigma-0's are known to have calm winds $(<2 \mathrm{~m} / \mathrm{s})$ [8]. Finally, the percentage of negative sigma- 0 over the ocean was consistent with that obtained from prelaunch end-to-end simulations.

Another consistency check of the sigma-0 data was to plot the sigma- 0 difference between neighboring cells over land versus orbit step for each antenna. This plot was generated by averaging over many orbits and many cells at the same latitude, and it was anticipated that the sigma- 0 differences over land should be well behaved and continuous. A typical plot of the result is shown in Fig. 11 and indeed, the sigma- 0 differences are reasonably behaved.

A quick check of the sigma-0 products over ocean can be performed by plotting the histogram of sigma- 0 over ocean, using incidence angle as a free parameter. A typical plot is shown in Fig. 12. For very low values of sigma-0, the density function has a second peak. It turns out that sigma-0 drops off rapidly at low wind speeds and consequently produces a large number of near-zero sigma- 0 values for Rayleigh distributed wind speeds.

\section{SENSOR CALIBRATION}

In order to retrieve wind vectors to the desired accuracy as specified by the science requirements [6], we needed to calibrate NSCAT and to compute sigma- 0 with a high degree of accuracy. The calibration of the instrument electronics was discussed in Section VI-B, and the verification of the 

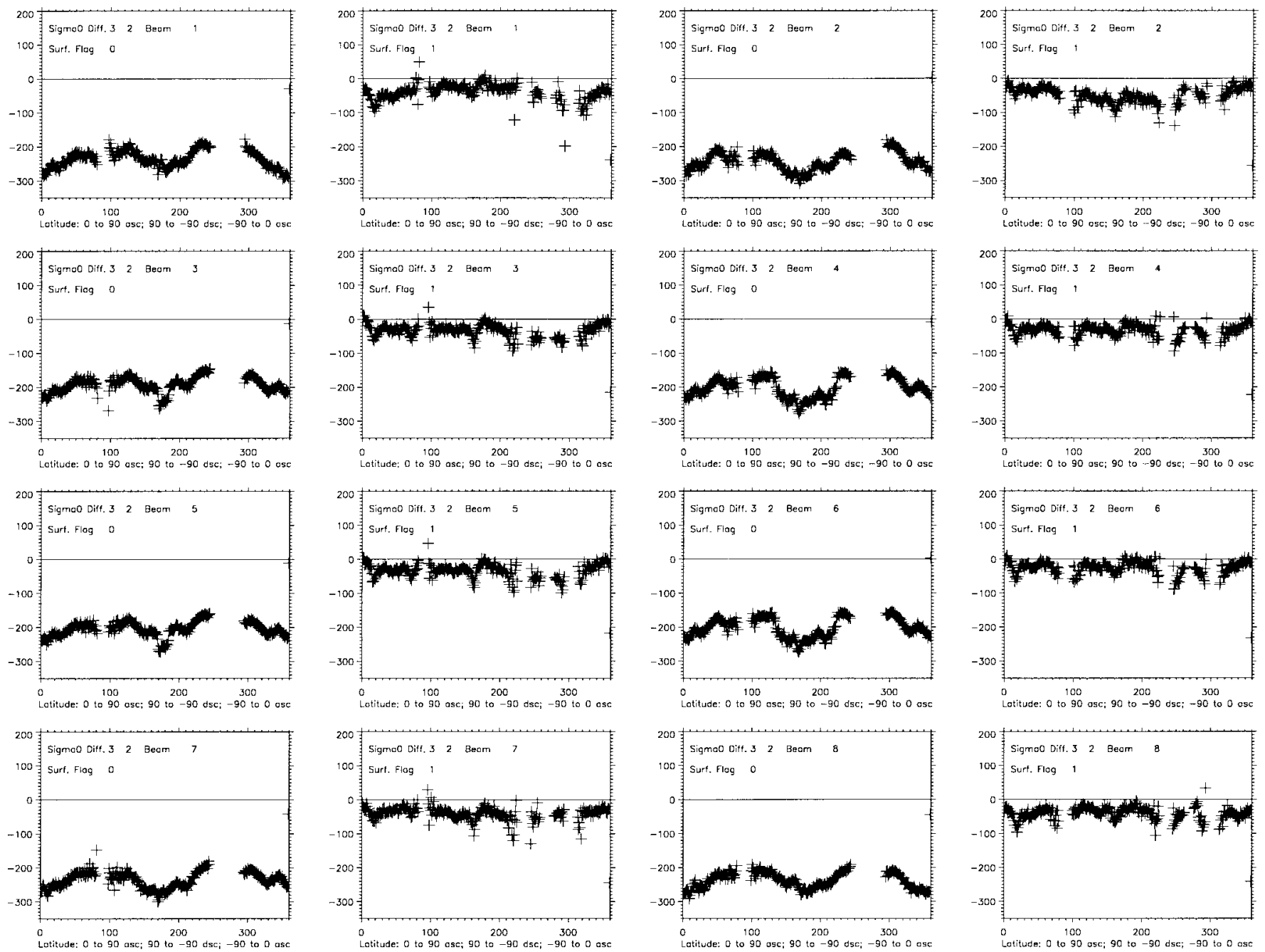

Fig. 11. Neighboring-cell sigma- 0 difference over ocean and land. The sigma- 0 variation is larger over the ocean, since sigma-0 is sensitive to wind speed and direction. However, over land, we anticipate the variation to be small. The $y$-axis is the sigma- 0 difference in units of $0.01 \mathrm{~dB}$, and the horizontal axis is the orbital step number. The notation sigma-0 Diff. 32 means the difference in sigma-0 between sigma- 0 cells \#3 and \#2. Surface flag 0 means ocean, and surface flag 1 means either ice or land.

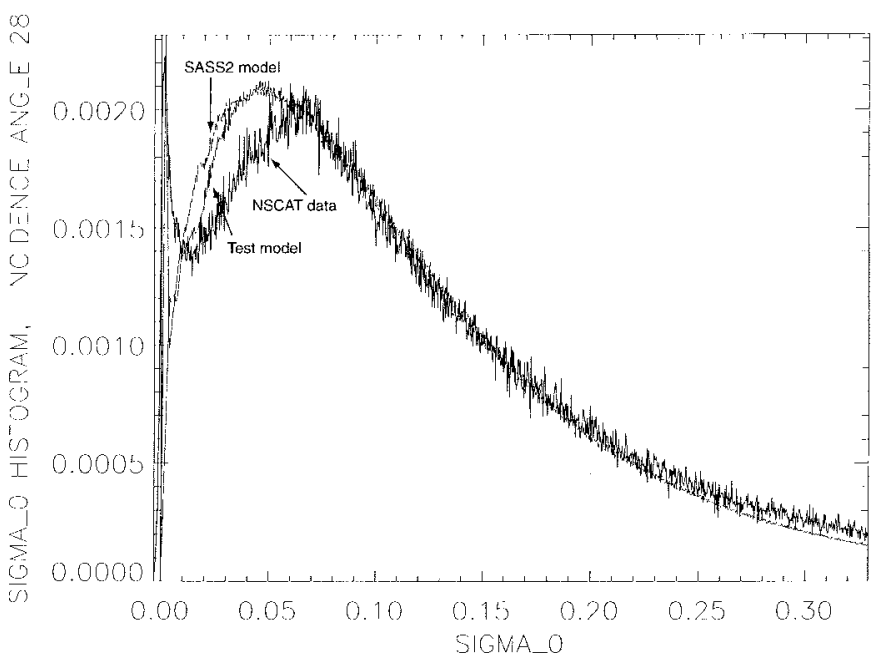

Fig. 12. Sigma-0 histogram over ocean at $28^{\circ}$ incidence angle. The results from NSCAT are plotted against the plots using SASS-2 model function, and a test model by lowering the sigma-0 of the SASS- 2 model function at low wind speed $(<3 \mathrm{~m} / \mathrm{s})$ by $3 \mathrm{~dB}$. This figure is used to demonstrate that the second peak of the sigma- 0 histogram at very low sigma- 0 is due to the fact that sigma-0 at low wind speed (as measured from NSCAT) is lower than that predicted by SASS-2 model function. computation algorithms were discussed in Section VI-C. In this section, we will present the calibration of antenna beam pointing and the radiometric beam balance of all eight antenna beams.

\section{A. Beam Pointing Calibration}

There are a number of factors which affect the antenna beam pointing: antenna deployment angles, spacecraft attitude, and mechanical and thermal deformation. These factors are coupled and it is very difficult to verify them independently. The goal of the beam pointing calibration is to verify and calibrate the on-orbit beam pointing.

The prelaunch antenna deployment angles and coordinate definition had been verified with the antenna alignment cognizant engineer. A quick check on whether the antennas were fully deployed was performed by using the housekeeping engineering telemetry (the antenna latch indicator) and by comparing the locations of small islands with a detailed land map. For the latter, the agreement is within $25 \mathrm{~km}$, indicating the antennas were fully deployed. 

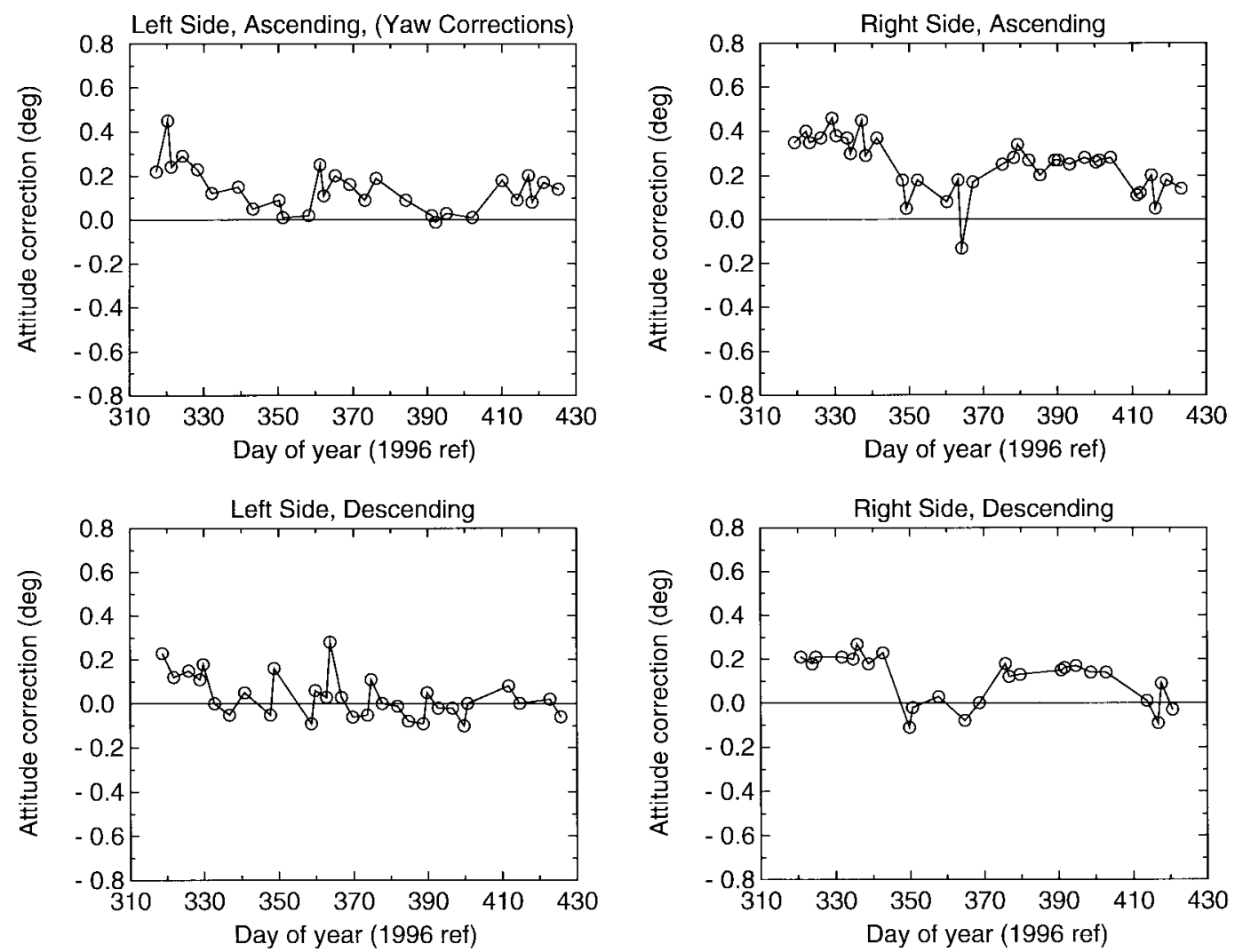

Fig. 13. Spacecraft (S/C) attitude determination using calibration ground station (CGS) measurements. The CGS measurements were segmented into S/C ascending and descending, and whether CGS was on the right or left side of the $\mathrm{S} / \mathrm{C}$ nadir track. The values plotted here are differences between CGS measurements and ADEOS reported yaw angle determination (from mission telemetry).

Three other methods were used to determine the antenna beam pointing: analysis of calibration ground station data, analysis of uniform natural target sigma-0 data, and independent spacecraft attitude determination using raw spacecraft housekeeping telemetry. The details of these methods will be discussed below. The major conclusion from these studies is that attitude determination errors of the order of a few tenths of a degree were likely to have existed [9]. Since there was no postlaunch verification of spacecraft attitude conducted by ADEOS, there is no reasonable way for us to improve this attitude determination uncertainty.

1) Beam Pointing Determination Using the Calibration Ground Station: For the period from October 1, 1996 through February 28, 1997, a calibration ground station (CGS) was deployed at JSC Western Test Facility located in White Sands, NM. It was capable of transmitting pulses to NSCAT and also capable of receiving radiation from NSCAT. For each NSCAT pass over the CGS site, a measurement was made of the antenna narrow beam pattern of the three antennas (fore, mid, and aft) on one side of the spacecraft nadir track. The timing of the peak gain of these three antenna beams can be used to estimate the spacecraft attitude (roll, pitch, and yaw), assuming that the antennas were deployed at the desired angles.

A sample plot of the yaw angle is shown in Fig. 13. We observe that the effective attitude differs from ascending node to descending node, and from left to right side of the nadir track. The magnitude of the variation is typically on the order of a few tenths of a degree. This is quite in contrast with the reported spacecraft attitude (see Fig. 14), which is typically a few hundredths of a degree in magnitude. As we will see in the next two subsections, this discrepancy persisted. Therefore, there were probably short-term spacecraft attitude knowledge errors of a few tenths of a degree, which would contribute to about a few tenths of a decibel in short-term sigma-0 variation.

2) Beam Pointing Determination Using Natural Targets: As part of the beam balancing described in the next section, natural targets such as the Amazon rain forest and central Russia were used to determine NSCAT beam pointing by computing the sigma- 0 difference (as a function of incidence angle) as measured by the different antenna beams and relating the difference to the spacecraft attitude [9]-[11].

Another method used to infer the accuracy of the spacecraft attitude determination was to compute the beam-to-beam bias over the open ocean, separating ascending and descending passes. This method also indicated similar ascending versus descending attitude variations as described earlier (see Section VI-B2 for more details).

3) Independent Attitude Determination by Post-Processing of Raw Housekeeping Attitude Data: The attitude data as reported by ADEOS came directly from the spacecraft without further ground processing. As an independent verification, JPL asked NASA Goddard Space Flight Center (GSFC) Flight Dynamics Division to independently determine the spacecraft 


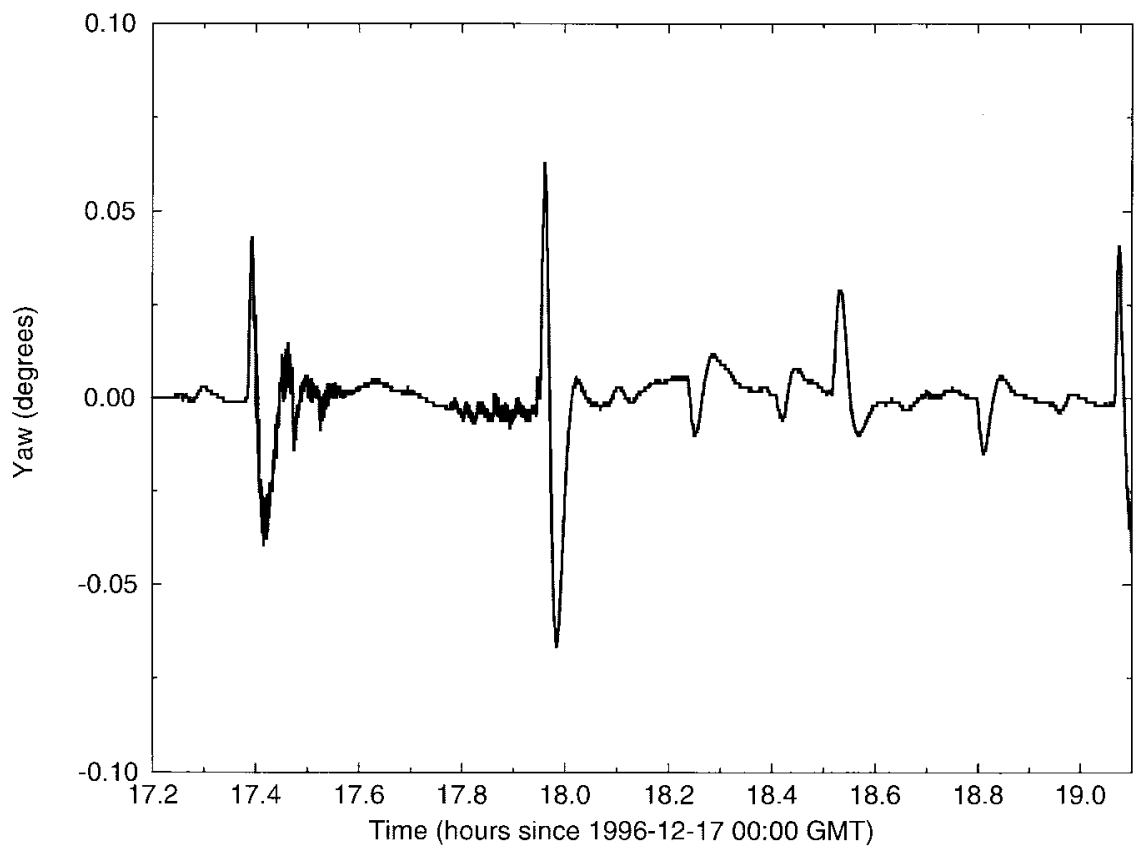

Fig. 14. Typical spacecraft yaw angle (as reported by ADEOS mission telemetry) as a function of orbit time.

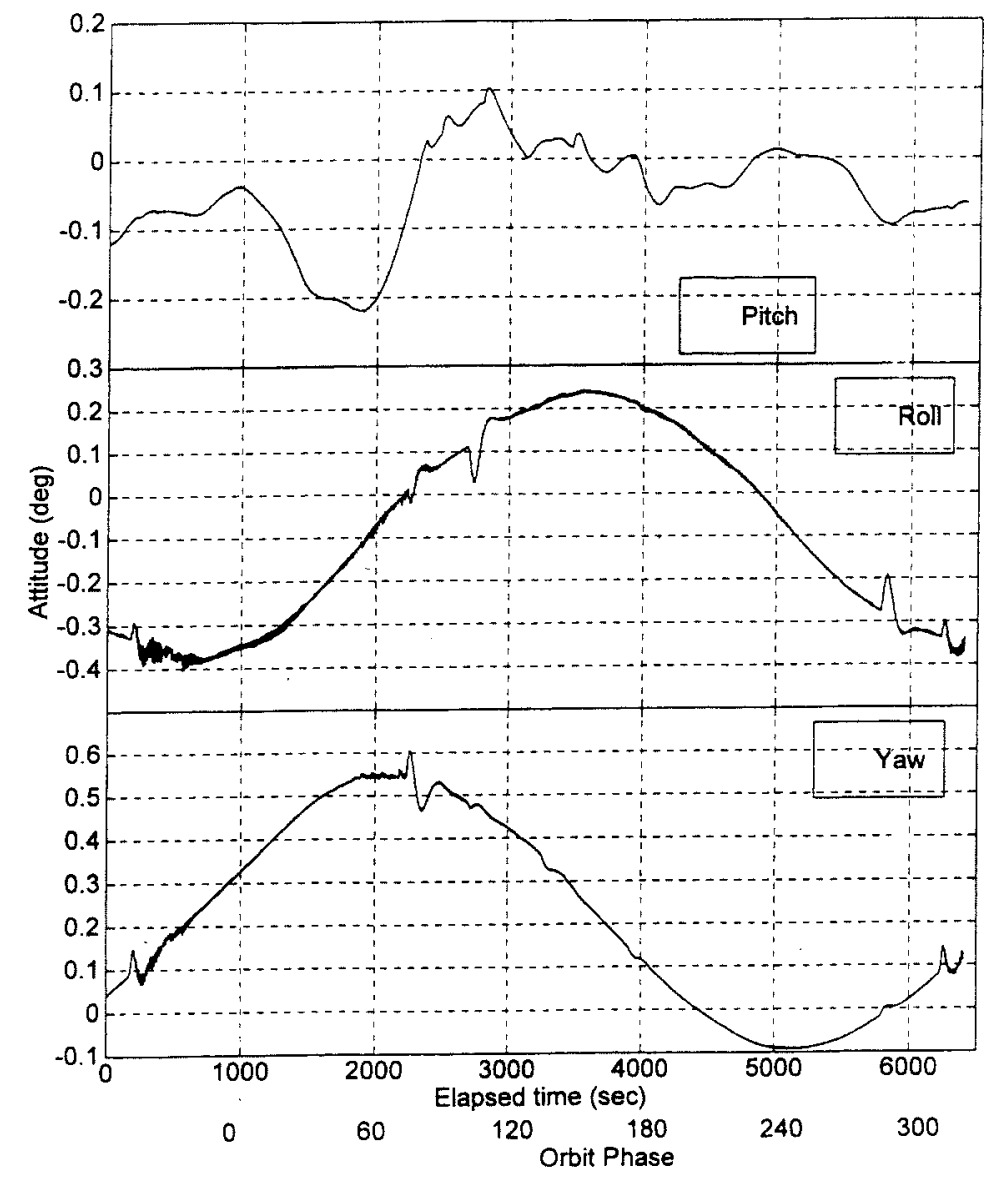

Fig. 15. Sample plot of the independent attitude determination using one-orbit of ADEOS raw attitude housekeeping telemetry. ADEOS CFADS attitude starting at 961129.234607.

attitude, via further analysis of the raw attitude housekeeping data [12].

Since ADEOS only downlinked one orbit (about $100 \mathrm{~min}$ ) of housekeeping data each day, and only for orbits near Japan, it is difficult to compare GSFCO's results with that of the
CGS or natural targets. Typical attitudes determined by GSFC are shown in Fig. 15. There we see that attitude variations of a few tenths of a degree were observed over one orbit. This is consistent with those reported in the two previous subsections. 


\section{B. Antenna Beam-to-Beam Radiometric Balancing}

NSCAT has eight antenna beams (six V-polarization and two H-polarization), four on each side of the nadir track (see Fig. 2). Since measurements from multiple beams are used together for wind retrieval, it is necessary to perform a radiometric beam balance of all eight antenna beams. There are three methods for determining the beam balance: analysis of sigma- 0 data over the open ocean, analysis of sigma- 0 over uniform land targets, and analysis of data from the CGS. In principle, the third method of using the CGS is the most direct and most accurate means of determining the beam balance. However, two factors caused us not to use the CGS-derived beam balance for data processing. Due to the nature of the CGS measurements, the CGS beam balance was very sensitive to spacecraft attitude determination errors. Beam balances derived from distributed target analyses absorbed the unknown attitude determination errors. Thus, although the CGS beam balance was potentially more accurate, the distributed target beam balance had the advantage of compensating for unknown attitude errors, in an average sense, when used to calculate sigma-0. Additionally, the CGS was not sufficiently stable from pass to pass, and this increased the errors in the beam balance derived from CGS data.

1) Beam Balancing Using Open Ocean Backscatter Measurements: Techniques for the relative calibration of scatterometer antennas using open ocean measurements and auxiliary information were developed and tested with ERS-1 data [13], [14] prior to the launch of NSCAT. Although they differ in detail, the open-ocean beam balancing approaches require accurate auxiliary knowledge of the distributions of wind speed and relative wind direction imaged by each of the antennas at each incidence angle, as well as reasonably accurate knowledge of the model function relating sigma0 to wind conditions and viewing geometry [13]. The use of open-ocean data is appealing, since large quantities of open-ocean backscatter measurements can be obtained rapidly, and the dynamic range of the measurements is precisely that encountered for actual wind measurements (in contrast with land and ice targets). It was shown in [13] that $0.2 \mathrm{~dB}$ relative accuracy could be obtained using as little as three weeks of scatterometer data.

The open-ocean beam balancing methods are not without drawbacks, however. Although precise prior knowledge of the model function is not required [13], systematic incidence angle dependent errors in the model function can result in absolute errors in calculated antenna gains as a function of incidence angle. While such sigma- 0 calibration errors will generally have insignificant effects on the accuracy of the retrieved winds (unless the prior model function is egregiously incorrect), they can seriously degrade the scientific utility of nonocean (land and ice) backscatter data [15]. In addition, the open-ocean techniques assume that the antenna calibration is stable over periods exceeding several months. Temporal variations in the antenna calibrations (e.g., as a result of satellite heating and cooling through the orbit cycle, or orbitposition-dependent attitude determination errors, as discussed in Section VII-A), prevent averaging of all ocean data, greatly complicate the calculations, and increase the time needed to acquire sufficient data to perform the beam balance analyses.

The initial open-ocean beam balancing analyses for NSCAT used the approach described in [13]. Operational global surface wind analyses from European Centre for Medium-Range Weather Forecasts (ECMWF) and the U. S. National Centers for Environmental Prediction (NCEP) were linearly interpolated in space and time to the locations of each NSCAT open-ocean sigma- 0 measurement. To eliminate contamination from sea ice and errors in the operational products at high latitudes, only ocean data equatorward of $52^{\circ}$ latitude in both hemispheres was used. As errors in the operational wind products are larger at very low and very high wind speeds, the analyses were further restricted to measurements for which the interpolated ECMWF wind speed was between 5 and 15 $\mathrm{m} / \mathrm{s}$. The SASS-2 model function was used initially, and a single, incidence angle dependent scale factor was determined (independent of orbit phase) for each antenna.

The relative antenna gain recalibration derived from this initial analysis resulted in significant improvements in the consistency and accuracy of the NSCAT vector winds and allowed a scientifically useful multimonth data set to be distributed to the international NSCAT Science Working Team by late November, 1996. However, the verification and validation activities using the calibration ground station and data from distributed nonocean targets demonstrated the presence of significant ADEOS attitude control knowledge errors. These errors were systematic with respect to orbit position and suggested that the accuracy of the backscatter measurements could be improved by calculating beam balancing scale factors which depended on orbit phase in addition to incidence angle. At the same time, ongoing model function refinement activities (summarized briefly below in Section VIII) showed that significant improvements over the prelaunch SASS-2 Ku-band model function were possible; as noted above and in [13], while the open-ocean beam balance calculation is relatively insensitive to model function errors, the use of an accurate prior model function improves the fidelity of the beam balance corrections.

The NSCAT open ocean beam balance approach was therefore extended and refined through integration with the model function refinement analysis. In particular, the empirical model function development approach [17], [25], based on direct collocations between NSCAT backscatter cross sections and wind velocity estimates derived from multiple operational global surface wind analyses (see Section VII below), allowed for the calculation of separate model function coefficients for ascending and descending tracks for each NSCAT beam. As the model function relating backscatter to surface winds and radar viewing geometry should be independent of orbit phase, differences in the empirical coefficients derived from ascending and descending data can be attributed to apparent time(or orbit-) phase dependent instrument calibration variations. Further, since antenna gain errors leading to beam imbalances are functions only of beam and incidence angle (and not variations of sigma- 0 within the measurement range), antenna calibration factors can be refined by examining only the $A_{0}$ model function coefficients (i.e., the azimuthally integrated 

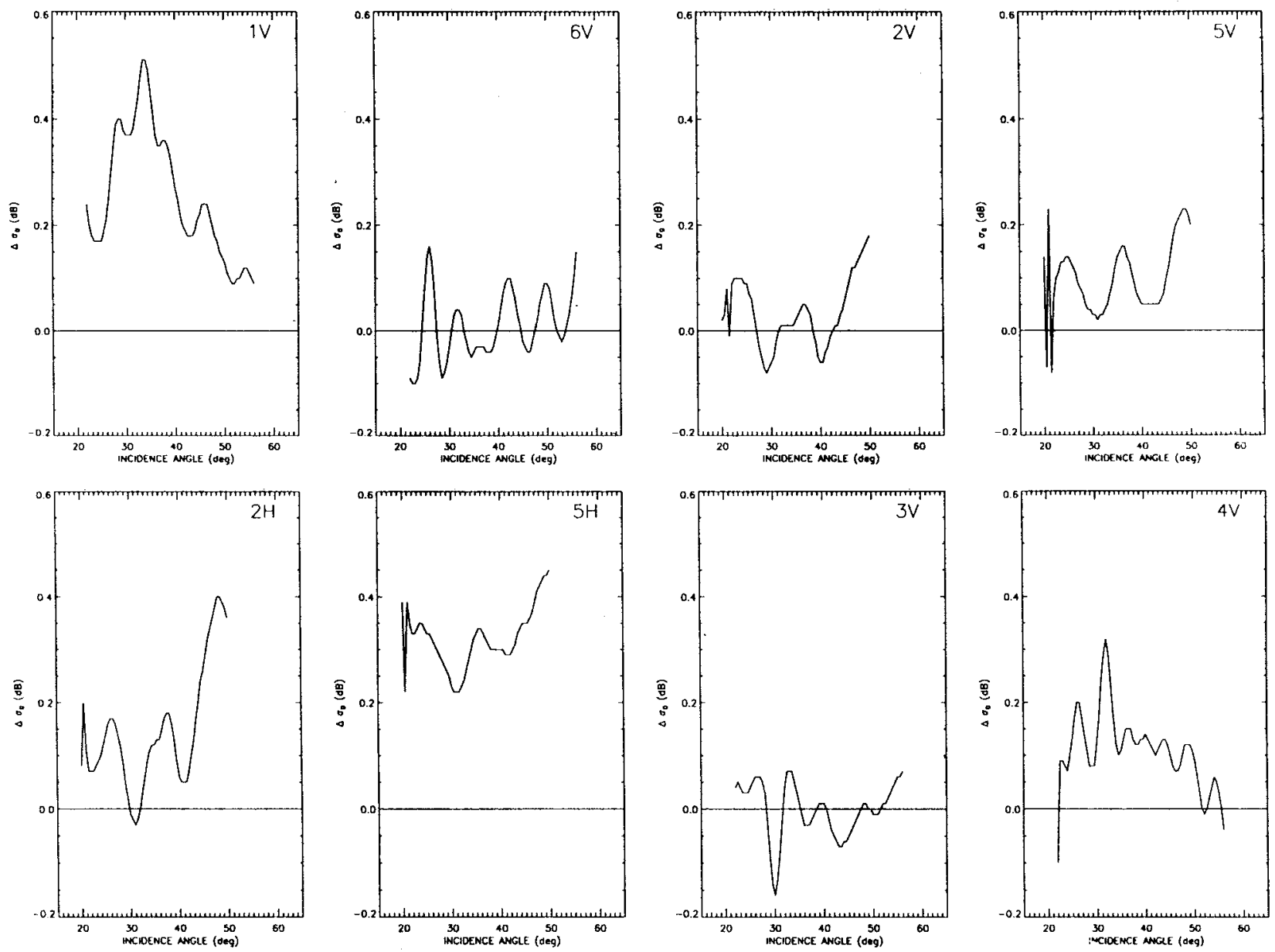

Fig. 16. Averaged ascending versus descending sigma- 0 measurement difference over open ocean. $(5-15 \mathrm{~m} / \mathrm{s}$, $\mid$ Lat $\mid \leq 52)$.

response which is a function only of incidence angle and wind speed) calculated from different beams and orbit phases.

Ascending versus descending beam balance differences for individual NSCAT antennas were determined by calculating a scale factor that minimized the integrated differences between empirical $A_{0}$ coefficients (the average sigma-0 over wind direction for a given wind speed) in the wind speed range $5-15 \mathrm{~m} / \mathrm{s}$ at each incidence angle. The results are shown in Fig. 16. The ascending/descending discrepancies have typical magnitudes of a few tenths of a decibel and are thought to result primarily from ADEOS attitude knowledge errors.

A final relative open-ocean beam balance was calculated from the following expression [28]:

$$
b_{I}(\theta)=\left\langle\bar{\sigma}(\theta)-\sigma_{I}(\theta)\right\rangle-\left\langle\bar{F}(\theta)-F_{I}(\theta)\right\rangle
$$

where the brackets \langle\rangle denote an average for the first three months of NSCAT measurements. The NSCAT measurement is denoted by $\sigma_{I}$, where the subscript $I$ denotes the antenna beam number. The model function value for the NSCAT measurement is denoted by $F$. The terms with the overbar denote an average over antenna beams. For v-pol (h-pol), this average is over the six v-pol (two h-pol) antennas. The model function term $F$ is evaluated using the collocated ECMWF wind speed and direction, and it is included to account for the fact that the mean wind speed and direction of the three-month averages are slightly different for the various antenna beams. The term is small and insensitive to the choice of the global circulation model (GCM) (i.e., NCEP versus ECMWF). An important property of the above equation is that the average of $b_{I}(\Theta)$ over the six v-pol antennas or the two h-pol antennas is zero. Thus, on the average, the beam balance correction does not modify the $\sigma_{o}$ versus $\Theta$ relationship. The correction only changes the relationship in a relative sense for each antenna beam. Beam balance corrections are computed separately for ascending and descending orbit segments, and the results are shown in Fig. 17 (with the results at high and low incidence angles being corrected using distributed land targets). With the exception of extreme (high and low) incidence angle regions, on-orbit beam biases calculated from the open-ocean technique were typically less than $|0.6| \mathrm{dB}$, demonstrating the essential accuracy of the extensive prelaunch antenna calibration efforts. Importantly, the final open-ocean beam balance for NSCAT incorporated both orbit phase dependent (ascending versus descending) factors and refinements in the Ku-band model function based on analysis of NSCAT data.

2) Beam Balancing Using Distributed Land Targets: Distributed land targets have been used for scatterometer beam balancing during SASS [18], [19] and ERS-1 [19] missions. This calibration method relies on large area homogeneous land targets with uniform azimuthal responses. For such 

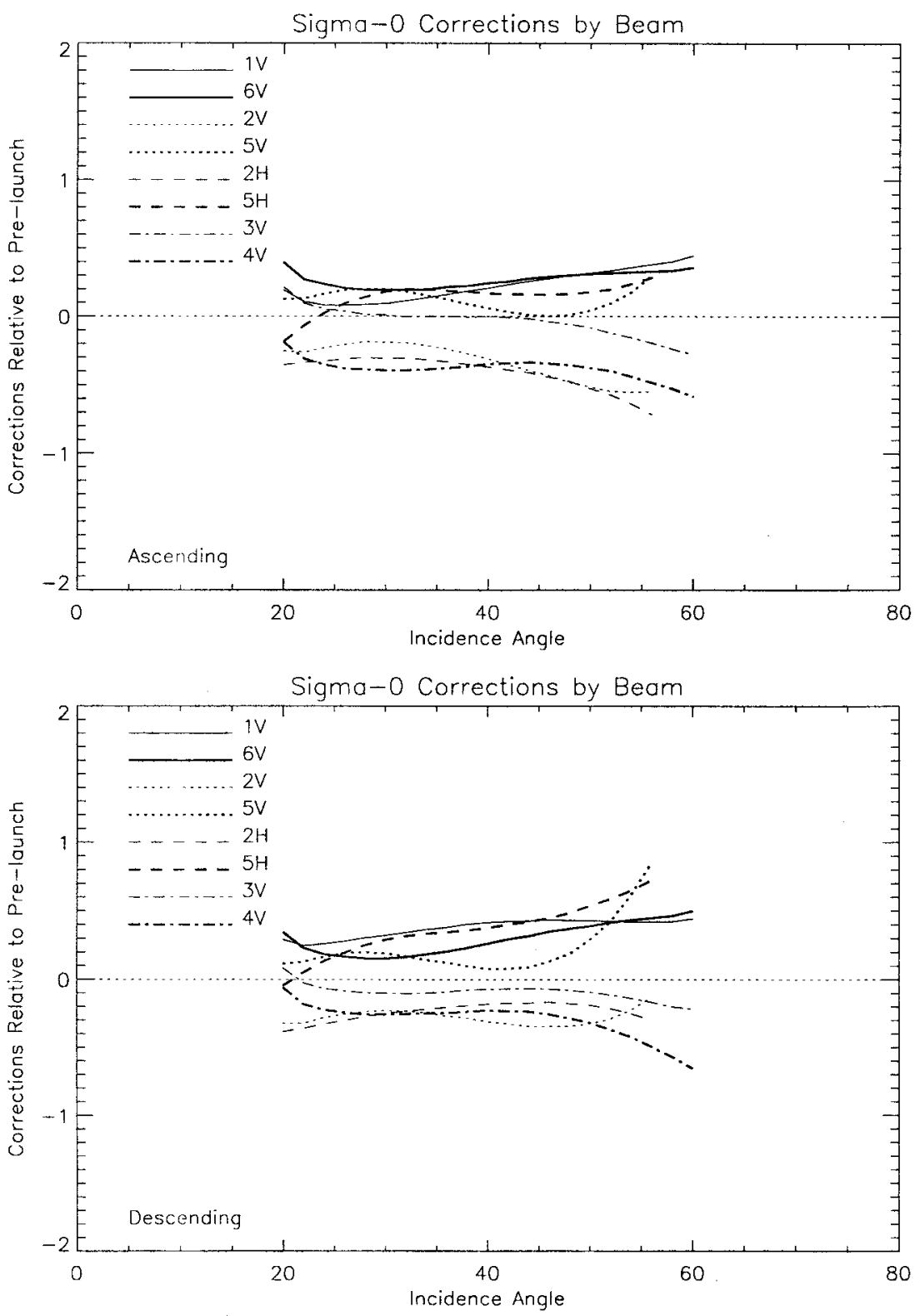

Fig. 17. Absolute antenna beam balancing results. The $y$-axis is the sigma-0 correction (in decibels) applied to each beam in order to balance the sigma-0 measurements.

targets, consistency is expected for all beams (azimuths) over a relatively short data set collection period for which temporal stability can be expected. The calibration set was selected by filtering data through high-resolution geographical masks. The masks were generated using the resolution enhancing scatterometer image reconstruction (SIR) algorithm [19], [20]. The Amazon rain forest is a traditionally used calibration target. It covers an area of approximately 3 million square kilometers. Its uniform and dense canopy makes the radar cross section independent of azimuth. The random orientation of individual scatterers in the vegetation canopy makes sigma0 essentially polarization insensitive [18], while the equatorial location minimizes seasonal effects. The observed diurnal sigma- 0 variation is within $0.5 \mathrm{~dB}$, with the highest sigma- 0 in the morning [18], [19]. In addition to the Amazon basin, another large area in central Russia was identified, during the calibration and validation (cal/val) activities, as being sufficiently homogeneous and isotropic. However, severe seasonal variations limited the use of Russian data to the snow-free period. Therefore, this data was less reliable than Amazonian data and was used only to independently confirm the beam balance calculated for the Amazon region.

While previous scatterometer calibration studies based on distributed land targets have generally used the entire large homogeneous area as a unit, during the NSCAT cal/val activities, the region was also subdivided into smaller location elements. This enabled testing of the homogeneity assumption, but increased the convergence time, because more time was required to collect enough data for reliable sigma-0 modeling in each location element. Results confirmed that introducing 


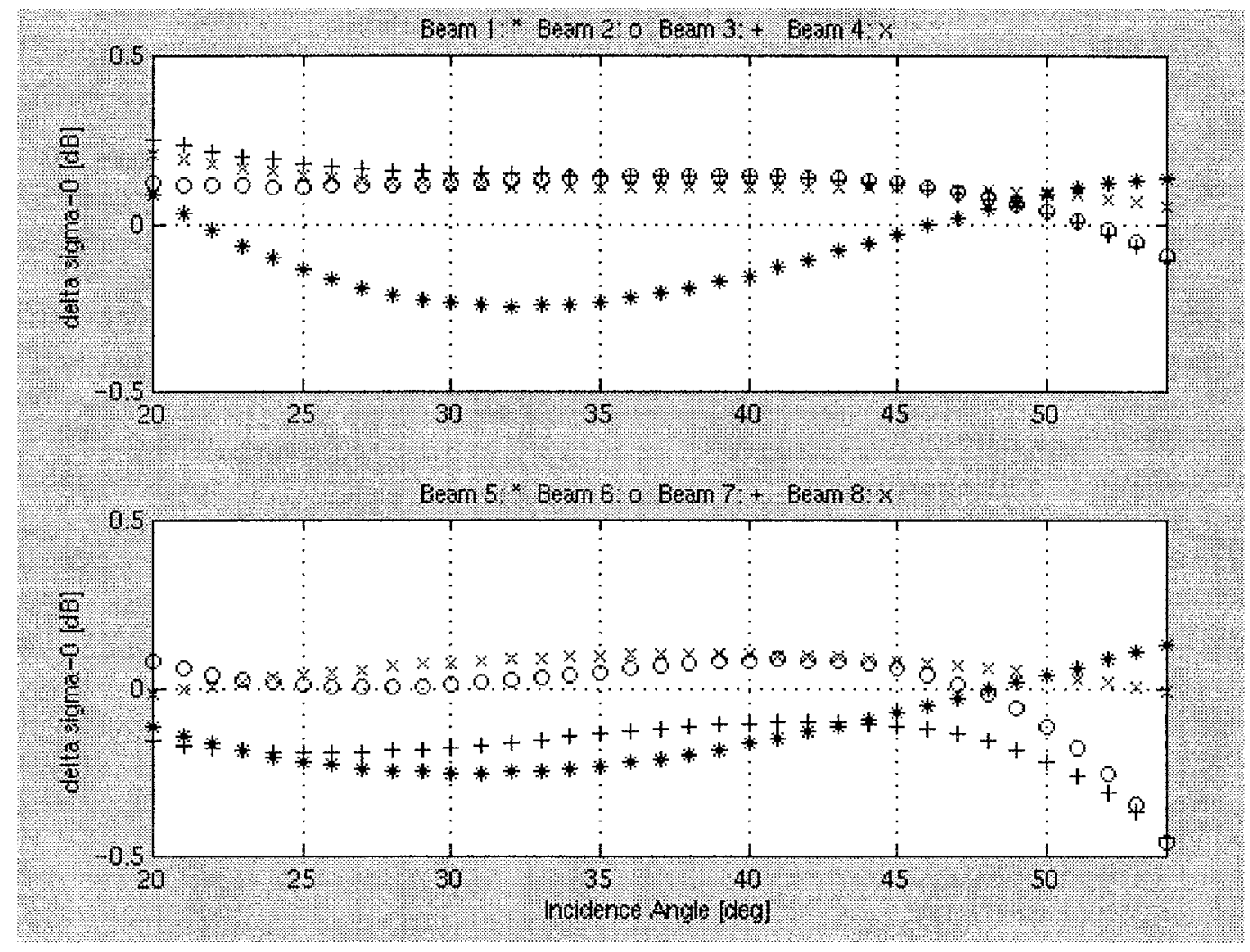

Fig. 18. Residual sigma-0 beam balance difference between ascending and descending passes.

spatial elements within the target does not change the beam balance compared to using the entire area as a single target.

The algorithm for computing antenna beam-to-beam sigma0 measurement bias is given below. Following Long and Skouson [19], a polynomial form is adopted to model the sigma- 0 response. Polynomial coefficients are estimated from measurements which have their corresponding integrated sigma- 0 cells completely within the mask. A value of mean sigma-0 for all antenna beams is obtained by averaging the individual beam responses. The beam-to-beam bias is calculated from the difference between the mean sigma- 0 response and the sigma- 0 response. This difference is added to raw measurements from each antenna beam. (Thus, the method is relative, aimed at removing biases among NSCAT beams.) The advantage of this method is its simplicity and its relatively fast convergence. Stable beam balance corrections are obtained using less than two-week data.

Beam bias corrections based on data from ascending passes are separated from those using descending pass data. The difference between the beam biases obtained from the ascending and descending passes is plotted in Fig. 18. The difference is on the order of few tenths of a decibel. This result is unexpected, since based on NSCAT antenna design, we anticipated that the beam balance would remain stable throughout the mission duration, independent of whether the satellite pass is ascending or descending. Further investigation suggested that this ascending versus descending imbalance could potentially be caused by an inaccurate attitude determination of a few tenths of a degree (see Section VII-A2). Taking the average of the ascending and descending beam biases, we obtained the overall sigma-0 correction for each antenna beam as shown in Fig. 19.

Finally, the beam bias results obtained from distributed land targets were compared to the beam balance corrections based on ocean data. Both correction sets were referenced to antenna $3 \mathrm{~V}$ to account for different references used by the two methods. Results are plotted in Fig. 17 for vertical polarization and for ascending and descending passes, respectively. We observe that there is generally good agreement between the results obtained from the two methods. Thus the distributed land target approach serves as an independent confirmation of the beam balance derived from the open ocean sigma- 0 measurements.

\section{Postlaunch Wind Verification and Validation}

The principal objective of the NSCAT instrument was the acquisition of all-weather, accurate, high-resolution measurements of near-surface wind speed and direction over the global oceans. Quantification of the accuracy of the NSCAT wind velocity data was therefore of primary programmatic and scientific importance. NSCAT postlaunch wind verification and validation involved three related, but distinct, activities: refinement of the Ku-band model function relating backscatter cross section to wind conditions and radar viewing geometry; identification of systematic errors in the NSCAT winds through comparisons between NSCAT data and selected statistics of operational surface wind analyses; and determination of the 


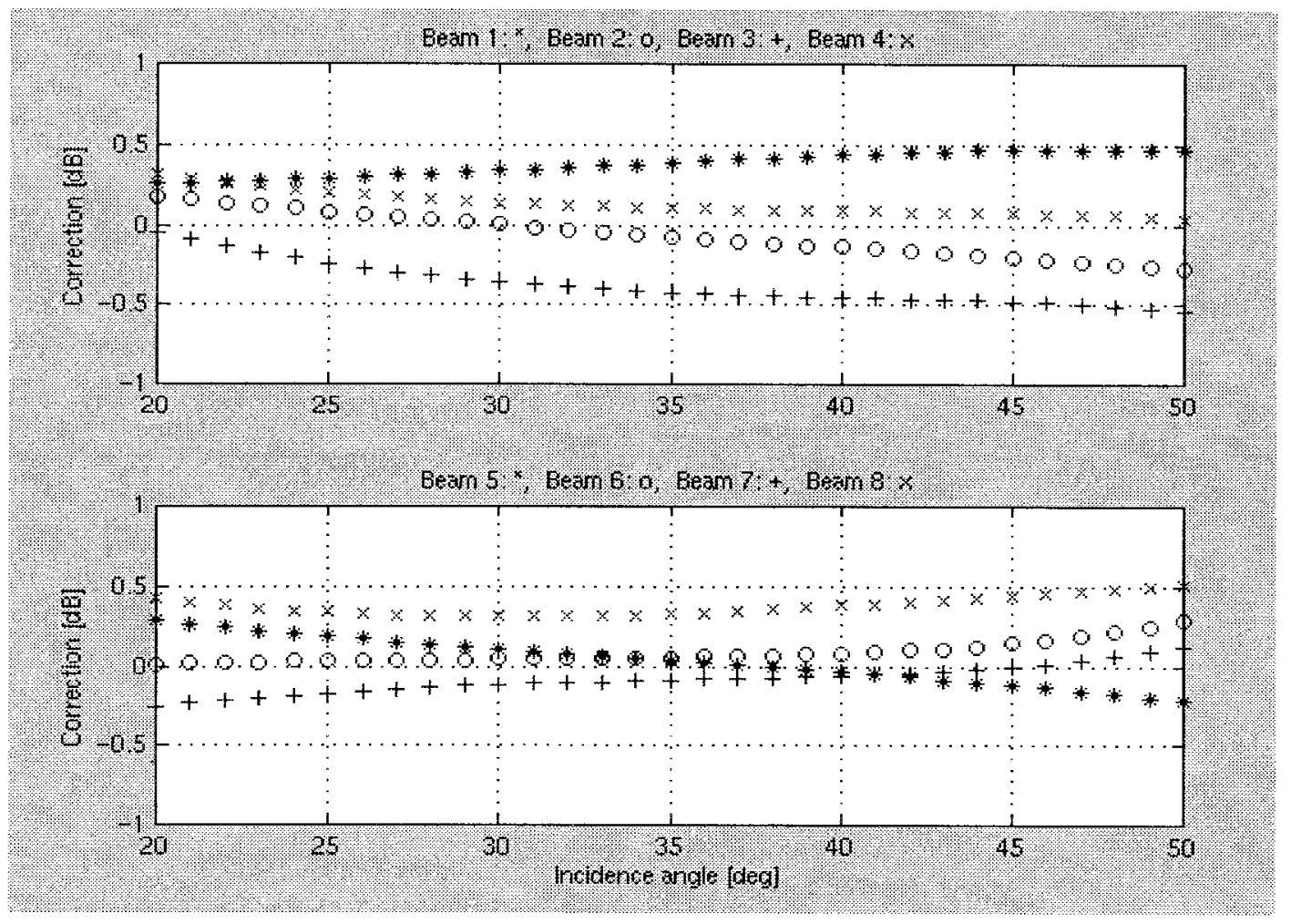

Fig. 19. Individual beam balancing corrections calculated from averaging ascending and descending passes.

wind speed and direction accuracy of the interim NSCAT vector wind data based on detailed comparisons with independent, high-quality, open-ocean buoy measurements. Brief summaries of the important approaches and NSCAT results are presented below; details of these activities that are beyond the scope of this paper can be found in the references.

\section{A. Ku-Band Model Function Development and Refinement}

Knowledge of the $\mathrm{Ku}$-band model function over the full range of wind and radar parameters is critical for the calculation of accurate wind velocities from NSCAT measurements [3]. Although increased understanding of the dynamics of wind-wave generation and radar scattering from realistic sea surfaces promises to allow future construction of fully analytic model functions, all operational satellite scatterometer model functions developed to date have been empirically based. For these model functions, adjustable coefficients are determined through analyses of in-situ measurements or statistics calculated from proxy data (such as surface wind velocities from operational numerical weather prediction global analyzes, or spatially and temporally collocated wind speeds from other satellite instruments). A principal difficulty with any empirical technique is the acquisition of comparison data spanning a sufficiently large range of conditions.

The SASS-2 empirical model function developed for the Seasat scatterometer [23], [24] was used to produce the preliminary NSCAT vector wind data set. Although the refined model outlined below addresses systematic errors in the SASS-2 model, the fact that vector winds retrieved from NSCAT data using the SASS-2 model came close to meeting the NSCAT science requirements showed both that SASS-2 properly incorporated the basic speed, direction, and incidence angle modulation of sigma-0 and that the NSCAT sigma-0 measurements were reasonably well calibrated in the absolute sense.

Wentz and Smith [25] and Freilich et al. [17] describe the approaches and results that led to the NSCAT-1 empirical model function. The method of [25] was based on comparisons between NSCAT and both collocated SSM/I and ECMWF measurements of surface wind speed, with an iterative procedure used to ensure that the resulting NSCAT wind speed histogram was smooth and matched that of the collocated ECMWF estimates. The approach of Freilich et al. ([15]; see also [14]) involved determination of the first six coefficients in the azimuthal Fourier series expansion of the model function for each wind speed, incidence angle, and polarization, through analysis of collocated and weighted surface wind estimates from both NCEP and ECMWF [16]. As refined for NSCAT, the method [17] explicitly corrected for coefficient biases resulting from random errors in the operational surface analysis products and the use of finite width bins in the coefficient calculation. Although the fundamental assumptions and methodologies underlying the two methods were different, the results were extremely similar, and the NSCAT-1 model function used to process the full data set used the Wentz and Smith mean $\left(A_{0}\right)$ coefficients and the Freilich et al. higher order directional modulations [25]. The operational NSCAT1 model function is used in the wind retrieval algorithms in tabular form (with sigma-0 given for each element of polarization, incidence angle, wind speed, and wind direction 
(a)

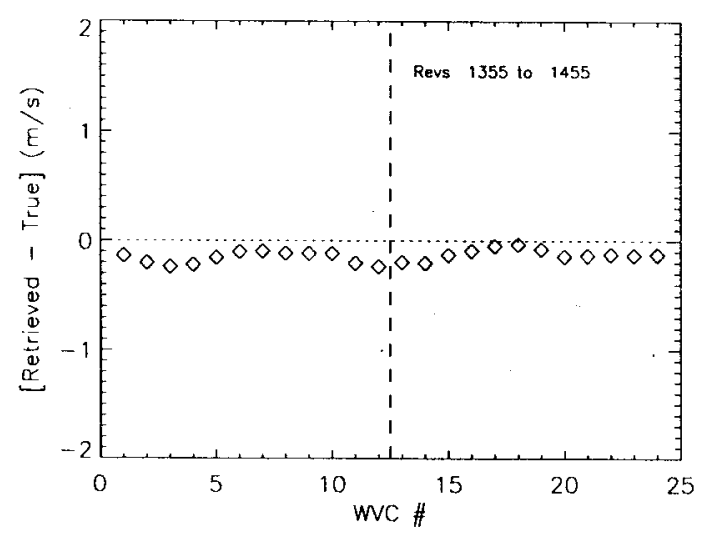

(c)

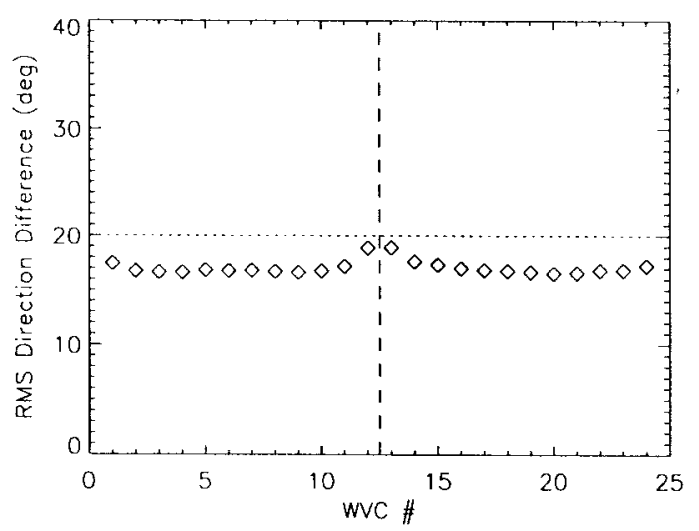

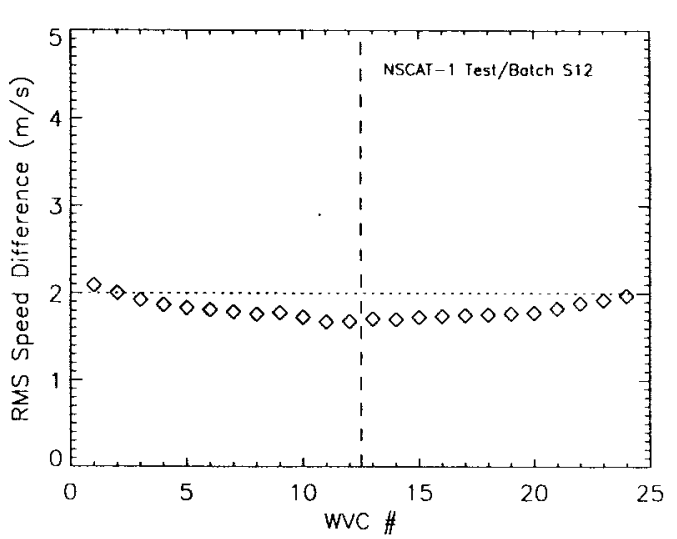

(b)

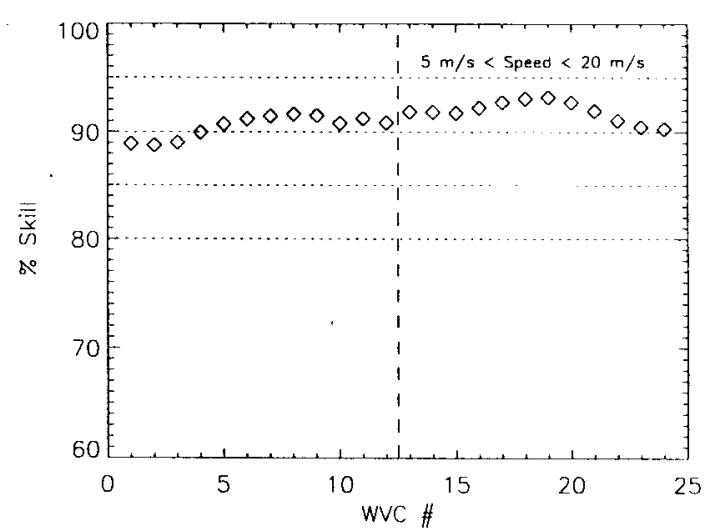

(d)

Fig. 20. Sample wind performance metric plots. The $x$-axis is the wind-vector-cell number (WVC \#), with each WVC center separated by $25 \mathrm{~km}$ using Doppler processing. (a) Speed bias, (b) rms speed error, (c) rms dir error, and (d) ambiguity removal skill.

parameter space). This approach decouples the precise form for the model function from the retrieval algorithms and allows modification of the model function without requiring changes to the processing software.

\section{B. Wind Product Verification}

Several recent works ([13], [17], [25], [26], and references therein) demonstrate that operational surface wind analyses correctly reproduce the low order statistics of the wind field on time scales from weeks to annual, although individual operational analyses may contain significant synoptic errors. The operational analyses are global, and thus rapidly provide a large quantity of comparisons with NSCAT data for qualitative validation and monitoring. NCEP and ECMWF operational analyses, spatially and temporally collocated with NSCAT measurements, were therefore used throughout the NSCAT postlaunch validation period to identify systematic errors in the NSCAT winds and to allow data sets retrieved with different model functions and beam balance corrections to be compared quantitatively. Fig. 20 shows differences between NSCAT and NCEP wind estimates, averaged over approximately six weeks of data, as a function of incidence angle for an intermediate version of the NSCAT model function. Speed bias, rms speed and direction errors, and estimates of ambiguity removal skill (defined as the fraction of NSCAT measurements for which the operational NSCAT processing selected the NSCAT vector closest in direction to the NCEP analysis direction) were found to be particularly useful statistics.
The wind speed density distribution generated using ECMWF has a regular Rayleigh distribution, independent of the cell location (see Fig. 21). The NSCAT wind direction distribution is also in reasonably good agreement with that of ECMWF, as shown by Fig. 22. This method is a powerful way to verify antenna beam balance and model function, since we believe that ECMWF wind fields, on the average, give a good overall wind speed and direction distribution. Any deviation from ECMWF wind speed and direction distribution is an indication that either the antenna beams were not correctly balanced or the model function in use needed some fine tuning. This was the case in the early stage of both the ERS- 1 and NSCAT missions. However, as shown in Figs. 21 and 22, after careful beam balancing and using the NSCAT-1 model function to process the winds, we obtained well behaved wind speed and direction density distributions.

Finally, to evaluate the accuracy of the ambiguity removal process, a new model-based QA algorithm was developed. This technique uses only NSCAT data and a simplified model of near-surface wind fields [21], [22]. The swath is segmented into overlapping $12 \times 12$ wind-vector-cell (wvc) regions and each region is classified according to estimated quality. Analyzing the nine-month NSCAT mission data set, the wind retrieval is at least $95 \%$ effective for $12 \times 12 \mathrm{wvc}$ regions with root-mean-square wind speeds of greater than $4 \mathrm{~m} / \mathrm{s}$ [22].

\section{B. Wind Product Validation}

NSCAT measured direction as well as speed, quantitatively, and the mission success required accurate direction as well 

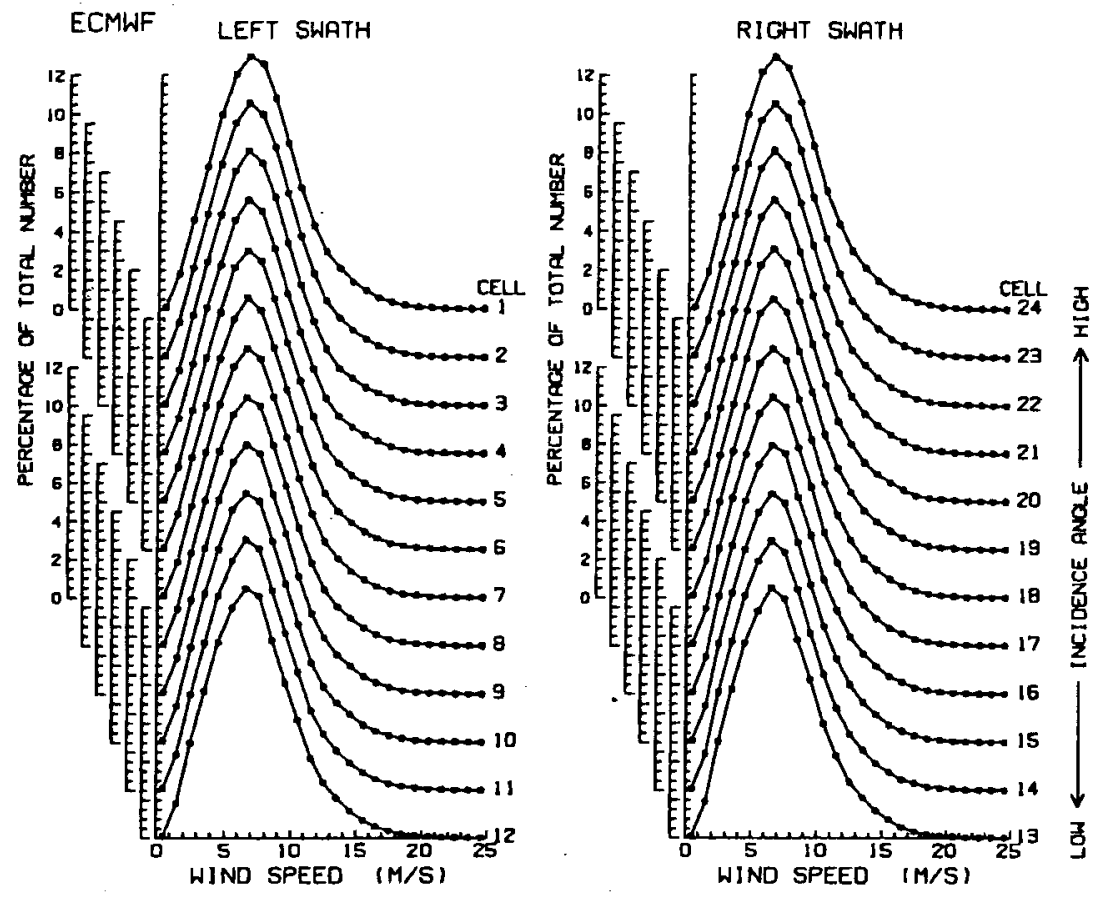

NSCAT-1 LEFT SWATH
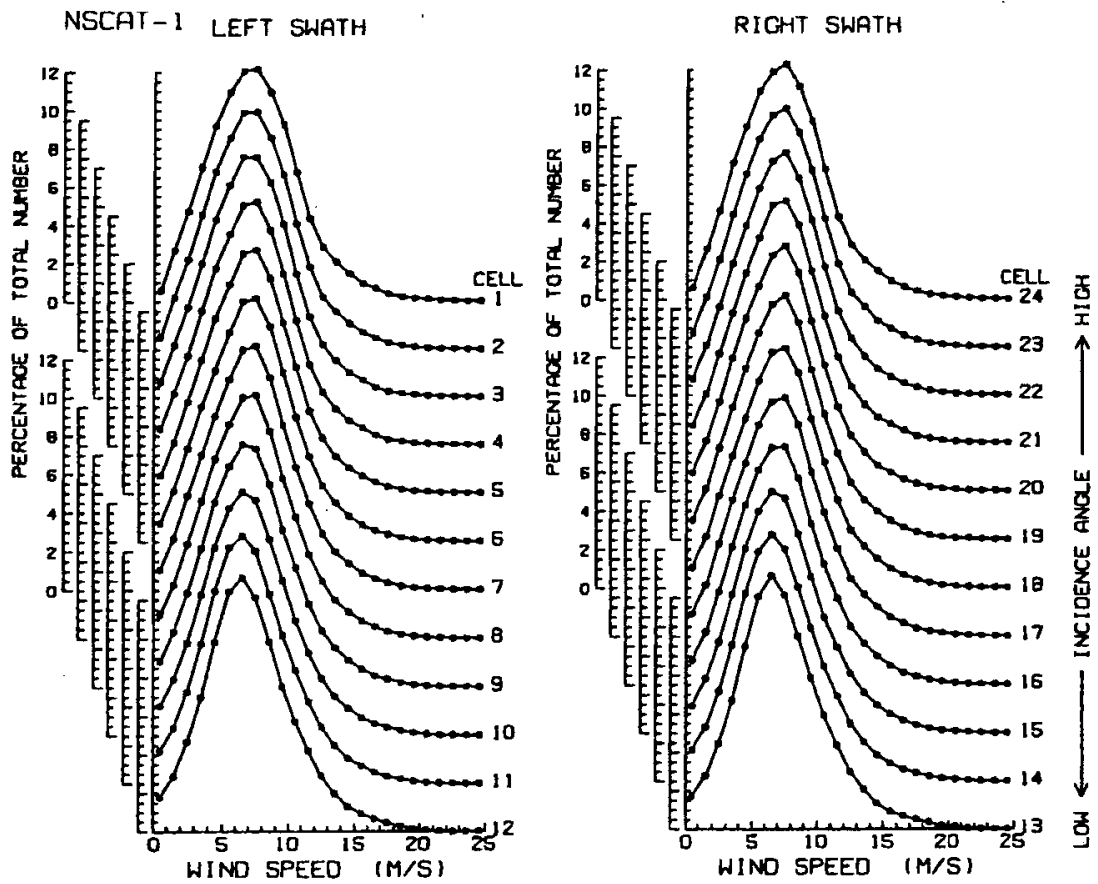

Fig. 21. Wind speed histogram comparing ECMWF and NSCAT wind measurements. The $x$-axis is wind speed in $\mathrm{m} / \mathrm{s}$. The $y$-axis is the percentage of the total measurements at a given wind-vector-cell.

as speed measurements. The overall accuracy of the vector wind measurements from NSCAT was further quantified by comparison with in-situ data from operational U.S. National Data Buoy Center (NDBC) ocean buoys. To validate the NSCAT wind products, 27 open-ocean moored buoys were used [27]. Fig. 23 shows the wind speed comparison between NSCAT and the NDBC buoys, for NSCAT observations within $50 \mathrm{~km}$ and $30 \mathrm{~min}$ of the reported buoy measurements. The analysis shows that the $25-\mathrm{km}$ resolution NSCAT wind speeds have unity gain, an offset (bias) of $-0.4 \mathrm{~m} / \mathrm{s}$, and an $\mathrm{rms}$ error of $1.15 \mathrm{~m} / \mathrm{s}$. For wind speeds greater than $6 \mathrm{~m} / \mathrm{s}$, the rms directional difference was found to be less than $17^{\mathrm{C}}$ (after discarding the approximately $3 \%$ of data with significant ambiguity selection errors), and a mean clockwise bias of about $8^{\circ}$. Performing the same analysis using NSCAT-sampled ECMWF wind vectors gave a wind speed comparison with nonunity gain and larger rms errors. The independent buoy validation gives a better picture of the true NSCAT accuracy 
(a)

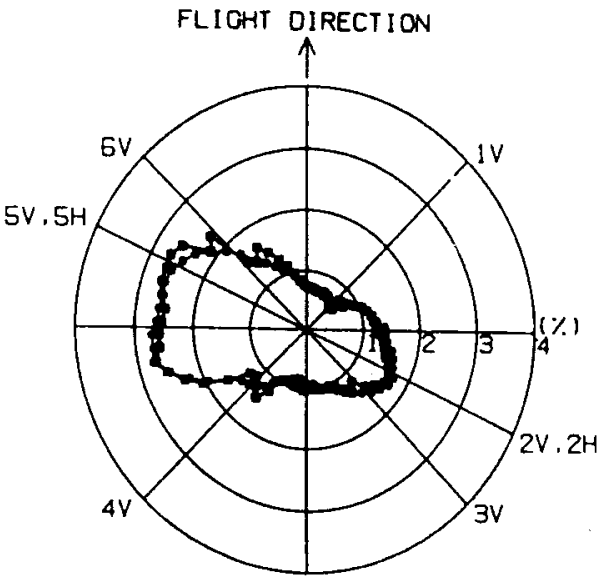

(c)

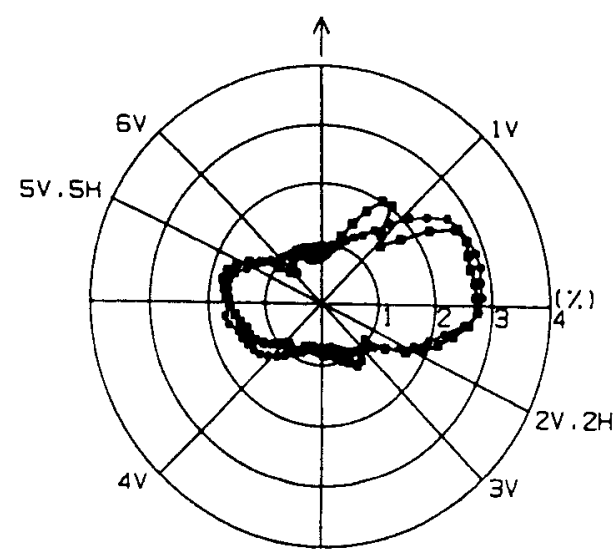

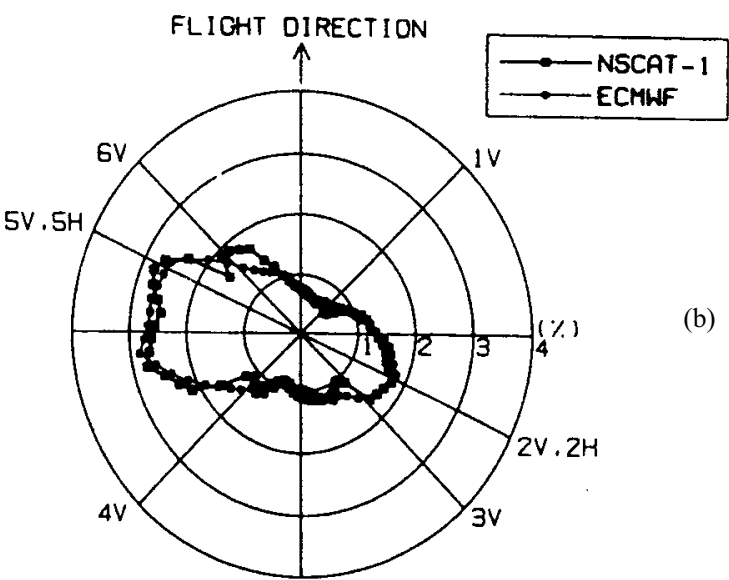

(b)

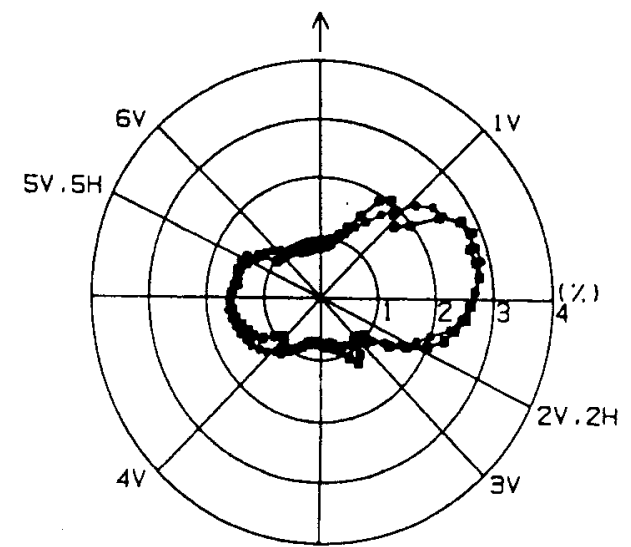

(d)

Fig. 22. Wind direction histogram comparing ECMWF and NSCAT wind measurements. If the wind direction histogram obtained from NSCAT agrees with that of ECMWF, the solid dot and the solid square will be on top of each other. The comparison between the ECMWF and the NSCAT wind direction histogram is a good way to test whether there is significant "error" either in the construction of the model function or in the computation of sigma-0. (a) Left swath ascending, (b) right swath ascending, (c) left swath descending, and (d) right swath descending.

than was obtainable from the numerical weather product (NWP) comparisons alone, and allows us to separate the NSCAT errors from the numerical model errors more clearly.

The NDBC buoy comparisons clearly demonstrate that NSCAT met its performance requirements of $2 \mathrm{~m} / \mathrm{s}$ speed $\mathrm{rms}$ (for speeds $3-20 \mathrm{~m} / \mathrm{s}$ ) and $20^{\circ}$ direction rms for the closest wind vector ambiguity.

\section{Summary AND CONCLUSIONS}

In this paper, we have presented the approaches and the analyses performed on the sigma- 0 and wind products to assure that they are reasonably behaved.

The verification and calibration of NSCAT is achieved using the following steps:

1) instrument functional and performance verification;

2) science data processing system verification;

3) sensor calibration;

4) construction of a geophysical model function;

5) wind products verification and validation.

The key conclusions are as follows.

1) The NSCAT instrument was functioning properly. All functional parameters, such as voltages, currents, and temperatures, are within the ranges predicted by prelaunch test data.

2) The instrument electronics were very stable. The temperature variation of the RF electronics over one orbit was less than $2{ }^{\circ} \mathrm{C}$. This, together with the temperature compensation loop for the receiver gain, provides a very stable receiver gain (to within $0.05 \mathrm{~dB}$ ) over a long period of time. The transmit power, as measured by the transmit power monitor, was very stable (to within 0.1 $\mathrm{dB})$. The frequency responses of the crystal filters are also very stable, and their shapes agreed very well with prelaunch measurements.

3) All of the sigma-0 computation algorithms were thoroughly checked and verified. The results passed the following two most severe tests: the continuity of sigma- 0 across channel boundaries and the locations of negative sigma-0's on the earth.

4) Based on our analysis of CGS data and sigma-0 data from natural targets, and also from an independent analysis of ADEOS housekeeping telemetry, the spacecraft attitude as reported by ADEOS may be off by a few tenths of one degree. This, in turn, affects the sigma- 0 measurement accuracy by a few tenths of one decibel. 


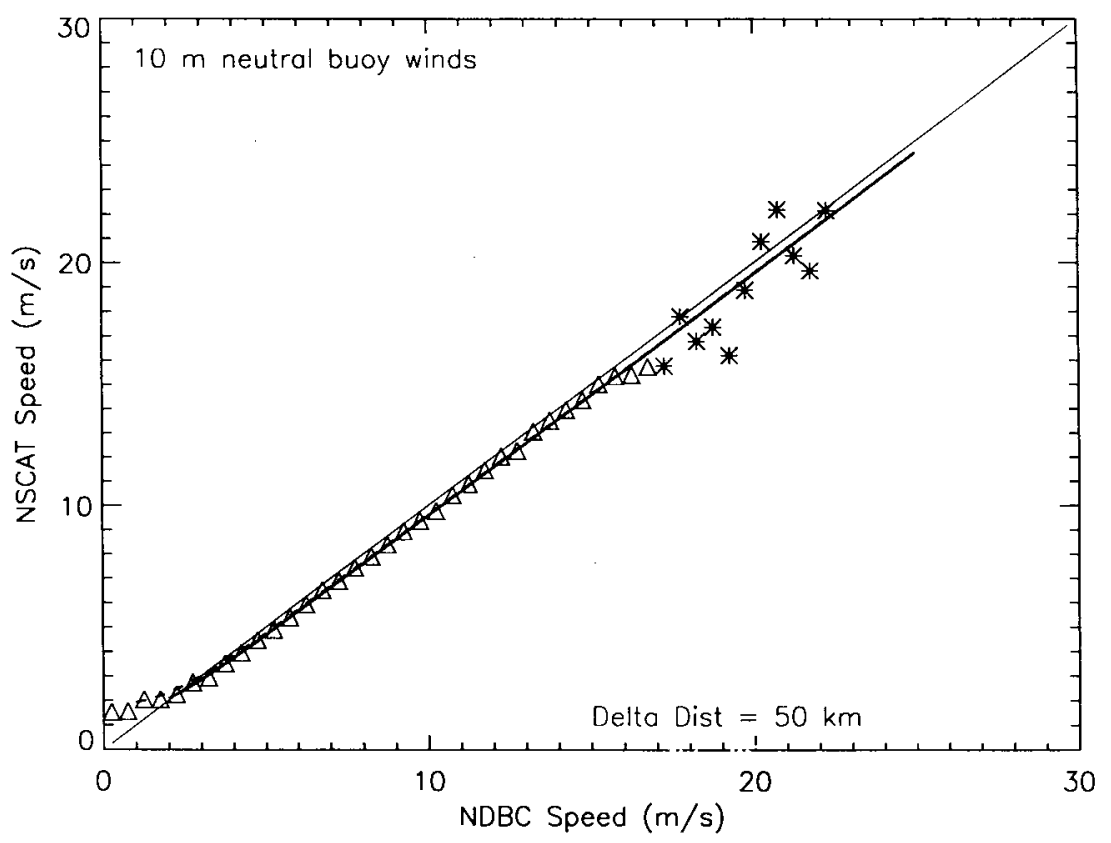

Fig. 23. Comparison of NSCAT wind speed with NDBC buoy measurements. The $\Delta$ symbols represent wind speed averages of 100 or more buoy measurements and the $*$ symbols represent wind speed averages of 5-99 buoy measurements. NSCAT-1 $\left(\rho^{2}>1.6\right)$.

5) The beam balancing was determined primarily by using sigma-0 data from the open ocean, with small incidence angle regions affected by land target analysis. The relative sigma- 0 accuracy of this method is estimated to be better than $0.3 \mathrm{~dB}$.

6) The initial wind products (processed using the SASS2 model function) exhibited some anomalous speed distributions at low wind speed and spikes in the wind direction distribution for certain wind directions. However, these problems were resolved by using the NSCAT1 model function to process the sigma- 0 data to winds.

7) The comparison of NSCAT wind vectors with those measured by NDBC buoys indicates that the wind speed and direction accuracy of NSCAT meets the science requirements.

\section{ACKNOWLEDGMENT}

The spacecraft for housing NSCAT was provided by National Space Development Agency (NASDA) of Japan. NSCAT raw data were collected and distributed to JPL by the Earth Observation Center of NASDA.

This postlaunch verification and calibration tasks were performed by a team of engineers from JPL and NSCAT Science Team members. Aside from the authors of this paper, the following individuals also contributed to various portions of this paper: M. Spencer, Dr. S. Yueh, Dr. S.-h. Lou, and G. Neumann contributed to the instrument and ground processing verification and calibration tasks, Dr. V. Hsiao contributed to some of the analysis sigma-0 products, Dr. W. Poulsen was involved in the instrument operation and routine monitoring of the instrument, J. Granger and Dr. R. West were involved in the operation and analysis of calibration ground station data, and Prof. N. Ebuchi provided the wind speed and direction density distribution results.
Finally, the authors wish to thank $\mathrm{H}$. Boettger, T. Hollingsworth, and J. Henessey for their generosity and help in providing the operational ECMWF surface wind analyses, within $36 \mathrm{~h}$, throughout the entire NSCAT mission.

\section{REFERENCES}

[1] Committee on Technical Assessment of Space Activities Commission of Japan, "Explanatory report on the termination of operation of ADEOS," NASDA, Sept. 14, 1997.

[2] T. Liu, Ed., Proc. NASA Scatterometer Science Symp., Maui, HI, Nov. 10-14. 1997.

[3] F. Naderi, M. H. Freilich, and D. G. Long, "Spaceborne radar measurement of wind velocity over the ocean-An overview of the NSCAT scatterometer system," Proc. IEEE, vol. 79, 850-866, 1991.

[4] S.-h. Lou and S. Yueh, "NSCAT K-factor report," JPL Internal Document, JPL Rep. JPL D 12435, Feb. 1995.

[5] J. Huddleston, "Analysis of the receiver gain of NSCAT," JPL Internal Document, JPL IOM 3347-94-12435, Sept. 9, 1994.

[6] NASA Scatterometer Mission Requirements, JPL Internal Document, JPL D-2676, Rev. A, July 1998.

[7] D. G. Long, "Sensitivity analysis of the NSCAT K-factor: Initial results," MERS Tech. Rep. MERS 97-006, Brigham Young Univ., Provo, UT, Dec. 5, 1997.

[8] W. J. Pierson, M. A. Donelson, D. G. Long, and W. B. Sylvester, "The measurement of calms, light air and light to moderate breezes by NSCAT," in Proc. NASA Scatterometer Science Symp., Nov. 10-14, 1997, pp. 80-88.

[9] D. G. Long, "ADEOS attitude determination from NSCAT measurements," in Proc. NASA Scatterometer Science Symp., Nov. 10-14, 1997, pp. $58-60$.

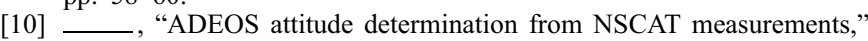
in Proc. Int. Geoscience and Remote Sensing Symp., Seattle, WA, July 6-10, 1998, pp. 654-656.

[11] J. Zec and W. L. Jones, "Effects of spacecraft attitude on the NASA scatterometer antenna calibration," in Proc. Int. Geoscience and Remote Sensing Symp., Seattle, WA, July 6-10, 1998 pp. 1998-2000.

[12] J. A. Hashmall, J. Landis, J. Sedlak, and R. Luquette, "ADEOS attitude determination and analysis," Final Rep., Computer Science Corp. and Goddard Space Flight Center to Jet Propulsion Laboratory, Jan. 31, 1998.

[13] M. H. Freilich, H. Qi, and R. S. Dunbar, "Scatterometer beam-balancing using open-ocean backscatter measurements," J. Atmos. Ocean. Technol., to be published. 
[14] A. Stoffelen, "A simple method for calibration of a scatterometer over the ocean," J. Atmos. Oceanic. Technol., to be published.

[15] J. Zec, D. G. Long, and W. L. Jones, "NSCAT sigma-0 biases using homogeneous land targets," AGU Oceans., to be published.

[16] M. H. Freilich and R. S. Dunbar, "A preliminary C-band scatterometer model function for the ERS-1 AMI instrument," in Proc. 1st ERS-1 Symp., 1993, vol. ESA SP-359, pp. 79-84.

[17] M. H. Freilich, H. Qi, and R. S. Dunbar, "A Ku-band scatterometer model function from NSCAT measurements and operational global surface wind analyses," J. Geophys. Res., to be published.

[18] I. J. Birer, E. M. Bracalente, G. J. Dome, J. Sweet, and G. Berthold, "Sigma-0 signature of the Amazon rain forest obtained from the Seasat Scatterometer," IEEE Trans. Geosci. Remote Sensing, vol. GE-20, no. 1, pp. 11-17, 1982

[19] D. G. Long and G. B. Skouson, "Calibration of spaceborne scatterometer data," IEEE Trans. Geosci. Remote Sensing, vol. 34, pp. 413-424, Mar. 1993.

[20] D. G. Long and P. Whiting, "Resolution enhancement of spaceborne scatterometer data," IEEE Trans. Geosci. Remote Sensing, vol. 31, no. 3, pp. 700-715, 1993.

[21] A. E. Gonzales and D. G. Long, "An assessment of NSCAT ambiguity removal," J. Geophys. Res., to be published.

[22] _ _ "A Quality assurance algorithm for NASA scatterometer wind ambiguity removal," in Proc. Int. Geoscience and Remote Sensing Symp., Singapore, Aug. 4-8, 1997, pp. 246-248.

[23] F. J. Wentz, S. Peteherych, and L. A. Thomas, "A model function for ocean radar cross-sections at $14.6 \mathrm{GHz}, " J$. Geophys. Res., vol. 89, pp. $3689-3704,1984$

[24] F. J. Wentz, L. A. Mattox, and S. Peteherych, "New algorithms for microwave measurements of ocean winds-Applications to Seasat and the special sensor microwave imager," J. Geophys. Res., vol. 91, pp. 2289-2307, 1986

[25] F. J. Wentz and D. K. Smith, "A model function for the ocean normalized cross section at $14 \mathrm{GHz}$ derived from NSCAT observations," J. Geophys. Res., to be published.

[26] N. Ebuchi, "Statistical distributions of wind speeds and directions observed by NSCAT," in Proc. NASA Scatterometer Science Symp., Nov. 10-14, 1997, pp. 149-151.

[27] M. H. Freilich and R. S. Dunbar, "The accuracy of the NSCAT-1 vector winds: Comparisons with NDBC buoys," J. Geophys. Res., to be published.

[28] F. Wentz, "NSCAT antenna beam balancing over the ocean," Remote Sensing Systems Tech. Rep. 112897, Nov. 1997.

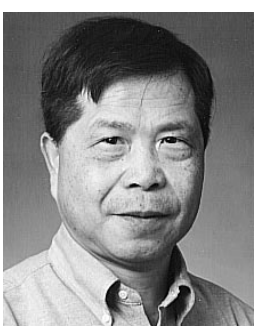

Wu-yang Tsai received the Ph.D. degree in theoretical physics from Harvard University, Cambridge, MA, in 1971.

He has performed research in high energy physics, classical and quantum electrodynamics, electromagnetic scattering, propagation and radiation, high-density plasma fusion, high-altitudenuclear-explosion (HANE) phenomenology, radiative heat transport, synthetic aperture radar, and currently on all aspects of scatterometer design and applications. He coauthored a graduate-level text book with Julian Schwinger et al. on Classical Electrodynamics. He joined the Jet Propulsion Laboratory (JPL), Pasadena, CA, in 1987 and was the Project Engineer of the EOS SAR preproject and the NSCAT project. He is currently the Project Engineer of SeaWinds on QuikSCAT (QSCAT) and Advanced Scatterometer Projects. He is also the Group Supervisor of the Scatterometer System Engineering Group in the Radar Science and Engineering Section of JPL.

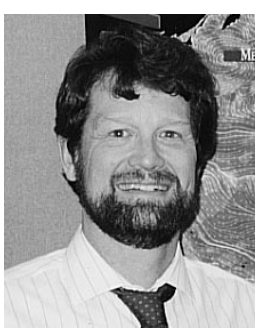

James E. Graf received the B.S.E. degree from Princeton University, Princeton, NJ, and the M.S. degree from Colorado State University, Fort Collins.

He has been employed in aerospace engineering activities since 1974. He is the Program Manager for the Jet Propulsion Laboratory's Scatterometer Program, Pasadena, CA. The program consists of the NASA Scatterometer (NSCAT), SeaWinds instruments, and the Quick Scatterometer Mission (QuikSCAT).
Carroll Winn, photograph and biography not available at the time of publication.

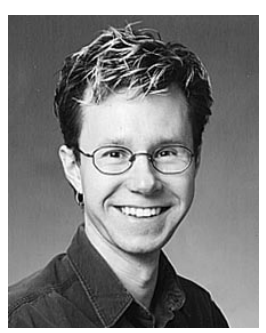

James N. Huddleston received the B.S. and M.S. degrees in electrical engineering from the Illinois Institute of Technology, Chicago, in 1990 and 1991, respectively.

In 1991, he joined the Radar Science and Engineering Section at the Jet Propulsion Laboratory (JPL), California Institute of Technology, Pasadena. There, he is the Sensor Verification Team Leader for the postlaunch calibration of QuickSCAT and also serves as the Cognizant Engineer for QSCAT Simulation and Performance Analysis.

Scott Dunbar, photograph and biography not available at the time of publication.

Michael H. Freilich, photograph and biography not available at the time of publication.

Frank J. Wentz received the B.S. degree in 1969 and the M.S. degree in 1971 both in physics from the Massachusetts Institute of Technology, Cambridge.

In 1974, he established Remote Sensing Systems, Santa Rosa, CA, a research company specializing in satellite microwave remote sensing of the earth. His research focuses on radiative transfer models that relate satellite observations to geophysical parameters, with the objective of providing reliable geophysical data sets to the earth science community. As a member of NASA's SeaSat Experiment Team (1978-1982), he pioneered the development of physically based retrieval methods for microwave scatterometers and radiometers. Starting in 1987, he took the lead on providing the worldwide research community with high-quality ocean products derived from satellite microwave imagers $(\mathrm{SSM} / \mathrm{I})$. He is currently a member of NASA Earth Observation System (EOS) Investigators Working Group, NASA Advanced Microwave Scanning Radiometer (AMSR) Team, NASA Scatterometer (NSCAT) Team, NASA Tropical Rainfall Mission (TRMM) Team, and NASA Pathfinder Activity. He has served on many NASA review panels and is a member of the American Geophysical Union. He is currently working on satellite-derived decadal time series of atmospheric moisture and temperature, the measurement of sea-surface temperature through clouds, and advanced microwave sensor designs for climatological studies.

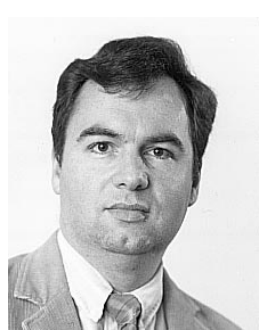

David G. Long (S'80-M'82-SM'98) received the $\mathrm{Ph} . \mathrm{D}$. degree in electrical engineering from the University of Southern California, Los Angeles, in 1989.

From 1983 to 1990, he worked for NASA's Jet Propulsion Laboratory (JPL), Pasadena, CA, where he developed advanced radar remote sensing systems. While at JPL he was the Project Engineer on the NASA Scatterometer (NSCAT) project. NSCAT was flown aboard the Japanese Advanced Earth Observing System (ADEOS) from 1996 to 1997 . He is currently an Associate Professor in the Electrical and Computer Engineering Department at Brigham Young University, Provo, UT, where he teaches upper division and graduate courses in communications, microwave remote sensing, radar, and signal processing. $\mathrm{He}$ is the Principle Investigator on several NASA-sponsored interdisciplinary research projects in remote sensing including innovative radar systems, spaceborne scatterometry of the ocean and land, and modeling of atmospheric dynamics. He is a member of the NSCAT Science Working Team. He has numerous publications in signal processing and radar scatterometry. His research interests include microwave remote sensing, radar theory, space-based sensing, estimation theory, computer graphics, signal processing, and mesoscale atmospheric dynamics. 


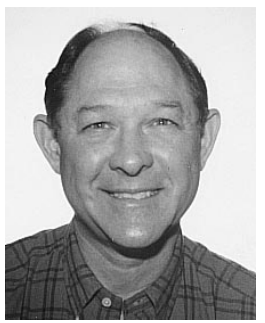

W. Linwood Jones (SM'69-F'99) received the Ph.D. degree in electrical engineering from the Virginia Polytechnic Institute and State University, Blacksburg, in 1971.

During his 25 years with NASA, he worked at Langley Research Center (1962-1981), NASA Headquarters (1988-1992), and Kennedy Space Center (1992-1994). At Langley, he managed the Microwave Techniques Research Section and later the Radar Remote Sensing Group. In 1975, he was NASA's Principal Investigator for the Seasat-A Satellite Scatterometer that demonstrated the proof of concept for inferring ocean surface vector winds. After a period working in private industry (1988-1992), he was a Satellite Program Manager for the NASA Headquarters where he was responsible for the following activities: Topex/Poseidon - radar altimeter/oceanography satellite; Tropical Rainfall Measuring Mission (TRMM) - rain/climate research satellite; and the NASA Scatterometer (NSCAT) - satellite instrument for ocean wind vector. From 1981-1988, he worked in the private aerospace industry as a Communications Subsystem Project Manager at the General Electric's Space Division in King of Prussia, PA. He is currently with the Central Florida Remote Sensing Laboratory, University of Central Florida, Orlando. 